OPEN ACCESS

Edited by:

Katsuhiko Tabuchi,

Shinshu University, Japan

Reviewed by:

Toru Yanagawa,

University of Tsukuba, Japan

Laura E. Maglio,

University of La Laguna, Spain

*Correspondence:

Danying Yang

yangdanying0315@126.com

Qingcui Zheng

zhengqingcui98@163.com

Specialty section:

This article was submitted to Neuroplasticity and Development,

a section of the journal

Frontiers in Molecular Neuroscience

Received: 02 July 2021 Accepted: 02 September 2021

Published: 29 September 2021

Citation:

Liu X, Ying J, Wang X, Zheng $Q$ Zhao $T$, Yoon $S$, Y $W$, Yang $D$, Fang $Y$ and Hua F (2021) Astrocytes in Neural Circuits: Key Factors in Synaptic

Regulation and Potential Targets for Neurodevelopmental Disorders. Front. Mol. Neurosci. 14:729273. doi: 10.3389/fnmol.2021.729273

\section{Astrocytes in Neural Circuits: Key Factors in Synaptic Regulation and Potential Targets for Neurodevelopmental Disorders}

\author{
Xing Liu ${ }^{1,2}$, Jun Ying ${ }^{1,2}$, Xifeng Wang ${ }^{3}$, Qingcui Zheng ${ }^{1,2 *}$, Tiancheng Zhao ${ }^{4}$, \\ Sungtae Yoon ${ }^{5}$, Wen $\mathrm{Yu}^{1,2}$, Danying Yang ${ }^{1,2 \star}$, Yang Fang ${ }^{1,2}$ and Fuzhou Hua ${ }^{1,2}$ \\ 'Department of Anesthesiology, The Second Affiliated Hospital of Nanchang University, Nanchang, China, ${ }^{2}$ Key Laboratory \\ of Anesthesiology of Jiangxi Province, Nanchang, China, ${ }^{3}$ Department of Anesthesiology, The First Affiliated Hospital of \\ Nanchang University, Nanchang, China, ${ }^{4}$ Mailman School of Public Health, Columbia University, New York, NY, \\ United States, ${ }^{5}$ Helping Minds International Charitable Foundation, New York, NY, United States
}

Astrocytes are the major glial cells in the brain, which play a supporting role in the energy and nutritional supply of neurons. They were initially regarded as passive space-filling cells, but the latest progress in the study of the development and function of astrocytes highlights their active roles in regulating synaptic transmission, formation, and plasticity. In the concept of "tripartite synapse," the bidirectional influence between astrocytes and neurons, in addition to their steady-state and supporting function, suggests that any negative changes in the structure or function of astrocytes will affect the activity of neurons, leading to neurodevelopmental disorders. The role of astrocytes in the pathophysiology of various neurological and psychiatric disorders caused by synaptic defects is increasingly appreciated. Understanding the roles of astrocytes in regulating synaptic development and the plasticity of neural circuits could help provide new treatments for these diseases.

Keywords: synaptic plasticity, astrocyte, glial transmitter, D-serine, glutamate, astrocyte-neuron signaling

\section{INTRODUCTION}

Astrocytes play an important role in terms of both function and their abundance in the central nervous system. For many years, astrocytes have been considered to play a supporting role in neural functions, such as ion homeostasis, regulation of local blood flow, clearance of neurotransmitters, and energy supply of neurons. In recent years, it has been found that astrocytes are also functional elements of "tripartite synapses" with presynaptic and postsynaptic neurons (Araque et al., 1999). Astrocytes control the formation, maturation, and plasticity of synapses through a variety of secretory and contact-mediated signals.

There are highly complex neural networks in the mammalian nervous system. Since a single neuron rarely performs a function on its own, it must establish connections with other neurons and form neural circuits through synapses. Therefore, the correct synaptic connection is the key to the formation of functional neural circuits. With the continuous production of immature synapses during development, some synapses with repeated inputs will become mature or stable, while other excess synapses will be eliminated or pruned (Clarke and Barres, 2013). Precise neural circuits are formed by initially overproducing neurons and synapses, followed by the elimination or pruning of superfluous neurons and synapses. 
Synaptic plasticity refers to the enhancement or weakening of long-term synaptic transmission related to activity, which is typically achieved through long-term potentiation (LTP) and long-term depression (LTD) (Yong et al., 2020). Synaptic remodeling occurs throughout life in diverse areas, prominently including the hippocampus, which is a structure essential for learning and memory consolidation (Nguyen et al., 2020). Synaptic morphological changes are associated with various neurodevelopmental disorders, including schizophrenia, autism spectrum disorders, and neurodegenerative diseases such as Alzheimer's disease and Parkinson's disease (Faludi and Mirnics, 2011; Li et al., 2016). Astrocytes participate in some pathophysiological processes of these diseases through the regulation of synapses and other effects. This review discusses the influence of astrocytes on the development of dendritic protrusions and synaptic plasticity during neural circuit development, while further deepening the understanding of the link between this influence and human neurodevelopmental disorders.

\section{THE CALCIUM SIGNAL OF ASTROCYTES PARTICIPATES IN THE TRANSMISSION OF INFORMATION BETWEEN ASTROCYTES AND NEURONS AND AFFECTS NEURAL ACTIVITY}

In contrast with neurons, which can produce action potentials, astrocytes are electrically non-excitable cells. Instead, theyGprotein-coupled metabolic receptors such as mevel to change the response of neurons (Cornell-Bell et al., 1990; Pasti et al., 1997). Therefore, Ca2 + signals are commonly measured as an indicator of astrocyte reactivity.

Research data shows that $G$ protein-coupled metabolic receptors, such as metabotropic glutamate receptors, type 1 cannabinoid (CB1) receptors, purinergic receptors, acetylcholine receptor (AchR), and GABAb receptors, or ligand-gated ion channel NMDA receptors (Kang et al., 1998; Papouin et al., 2017), are activated by corresponding physiological ligands. Hippocampal astrocytes in the region of the oriens layer of CA1 receive large amounts of cholinergic input and respond to acetylcholine released by synaptic terminals. The synaptically released $\mathrm{ACh}$ acts on $\mathrm{mAChRs}$, causing the release of internally stored $\mathrm{Ca}^{2+}$ (Araque et al., 2002). Indeed, astrocyte-neuron communication is complex. By using $\mathrm{Ca}^{2+}$ imaging and electrophysiology technology in hippocampal slices of mice, researchers found that Astrocytes can distinguish between cholinergic and glutamatergic synaptic activity and also between synaptic activity belonging to different axon pathways. Because glutamate released from alveus axons does not cause elevated $\mathrm{Ca}^{2+}$ levels as glutamate released from Schaffer collaterals and Ach released from alveus axons (Araque et al., 2002; Perea and Araque, 2005). This undoubtedly highlights the ability of astrocytes to integrate synaptic information in the astrocyteneuron communication.

Additionally, Phospholipase $\mathrm{C}$ can be activated to produce inositol IP3, which subsequently binds to specific receptors and induces the release of $\mathrm{Ca}^{2+}$ from the endoplasmic reticulum into the cytoplasm. The level of intracellular $\mathrm{Ca}^{2+}$ increases significantly, and a corresponding signal is transmitted in the form of $\mathrm{Ca}^{2+}$ waves. IP3-induced $\mathrm{Ca}^{2+}$ release is essential for initiating and maintaining $\mathrm{Ca}^{2+}$ waves between neurons and astrocytes. Most metabolic neurotransmitter receptors expressed in astrocytes activate phospholipase C/IP3 signal cascades. However, because the expression of IP3 type 2 receptor (IP3R2) in astrocytes is higher than in neurons, it leads to the depletion of stored $\mathrm{Ca}^{2+}$. The significant increase of intracellular $\mathrm{Ca}^{2+}$ is mainly induced by a combination of IP3 and IP3R2 in the endoplasmic reticulum (Hamada and Mikoshiba, 2020). This pathway is considered to be the basis of astrocytic $\mathrm{Ca}^{2+}$ waves, which are relatively slow calcium signals.

At the same time, the release of $\mathrm{Ca}^{2+}$ is amplified by the recruitment of the calcium-dependent endoplasmic reticulum $\mathrm{Ca}^{2+}$ channel, IP3 receptor (IP3s), and ryanodine receptor (RyR), which further lead to the increase of $\mathrm{Ca}^{2+}$ levels. Therefore, this is considered the main pathway that activates the calcium signal of astrocytes in the brain.

In addition to the fact that relatively slow $\mathrm{Ca} 2+$ waves can affect neuronal activity through astrocyte responses, transient changes of intracellular $\mathrm{Ca} 2+$ concentration $([\mathrm{Ca} 2+] \mathrm{i})$ in astrocytes can also regulate neuronal activity by inducing astrocytes to produce different responses (Araque et al., 2014). However, the reason why this transient $[\mathrm{Ca} 2+] \mathrm{i}$ is not regarded as the starting point for the activation of calcium signals in astrocytes in the brain is that the transient $[\mathrm{Ca} 2+] \mathrm{i}$ of astrocytes can be blocked without blocking neuronal transient $[\mathrm{Ca} 2+] \mathrm{i}$ or neuronal synaptic activity by blocking the abovementioned mGluRs and other different receptors by using specific receptor antagonists. In addition to these intracellular signaling pathways, it has been shown that the release of stored $\mathrm{Ca}^{2+}$ in cells downstream of G-protein-coupled metabolic receptors (also including other receptors) induces transient $\left[\mathrm{Ca}^{2+}\right]_{\mathrm{i}}$ in astrocytes. However, recent studies have challenged this concept because the cellular processes of astrocytes adjacent to neuronal synapses lack $\mathrm{Ca}^{2+}$ storage (Patrushev et al., 2013), which means that transient $\left[\mathrm{Ca}^{2+}\right]_{\mathrm{i}}$ cannot be produced through intracellular storage of $\mathrm{Ca}^{2+}$ in these astrocytes to affect the release of synaptic transmitters. Moreover, when IP3R2 was knocked out, the number of astrocytes with transient $\left[\mathrm{Ca}^{2+}\right]_{i}$ was greatly reduced, but the role of astrocytes in neuronal excitability (Petravicz et al., 2008), synaptic electrophysiology (Petravicz et al., 2008), and synaptic plasticity (Agulhon et al., 2010) was not affected. These studies show that the previous view that the effects of glial transmitters released by astrocytes on neuronal function are all caused by IP3-induced calcium release from the endoplasmic reticulum of astrocytes caused by transient $\left[\mathrm{Ca}^{2+}\right]_{i}$ needs to be further studied.

Indeed, different astrocyte processes produce transient $[\mathrm{Ca} 2+] \mathrm{i}$ at various times (Nett et al., 2002), and astrocyte processes with spatially localized transient $[\mathrm{Ca} 2+]$ i occur much more frequently than in somatic cells (Grosche et al., 1999; Nimmerjahn et al., 2009; Kanemaru et al., 2014). Furthermore, the advent of new techniques such as two-photon fluorescence imaging has made it easier to characterize different types of 
calcium transients in different parts of astrocytes. Transient $[\mathrm{Ca} 2+] \mathrm{i}$ in astrocyte processes can spread along the process into the soma (Nett et al., 2002; Kanemaru et al., 2014), and this signal transmission also occurs between cells (Nimmerjahn et al., 2009). The investigators introduced the $\mathrm{Ca} 2+$ chelator BAPTA into astrocytes in the dentate gyrus (Castro et al., 2011) and hippocampal area CA1 (Panatier et al., 2011) regio, causing an increase in the rate of synaptic failure. These studies invariably illustrate that increases of $[\mathrm{Ca} 2+] \mathrm{i}$ in astrocytes are essential for the regulation of neuronal and synaptic activity.

What's more, a recent study showed that although some transient $[\mathrm{Ca} 2+] \mathrm{i}$ astrocyte of were eliminated when IP3R2 was knocked out, this loss of store-release receptors did not have the anticipated major effect on astrocytes (Kanemaru et al., 2014; Srinivasan et al., 2015). In fact, a release of Ca2+ from internal stores is the main source of somatic transient $[\mathrm{Ca} 2+] \mathrm{i}$, but the transmembrane entry of $\mathrm{Ca} 2+$ in astrocytic processes, which may be mediated by endogenously active channels such as TRPA191 or receptor-gated Ca2+-permeable ion channels, produces a $30-40 \%$ elevation of $[\mathrm{Ca} 2+]$ i (Srinivasan et al., 2015). Therefore, Ca2 + signaling in astrocytes differs greatly between the soma and processes. Moreover, there are at least 8 times more transient $[\mathrm{Ca} 2+] \mathrm{i}$ in the processes than in the somata (Kanemaru et al., 2014; Srinivasan et al., 2015). Thus, previous conclusions based on knockdown of IP3R2 receptors actually did not adequately take into account the difference in $\mathrm{Ca} 2+$ responses between somatic cells and astrocytes, but the modulatory effects of astrocytes may be critical.

Some researchers have also proposed other pathways for the increase of $\left[\mathrm{Ca}^{2+}\right]_{\mathrm{i}}$ in astrocytes. For example, extracellular $\mathrm{Ca}^{2+}$ can flow into the cytoplasm due to the activation of $\mathrm{Ca}^{2+}$ permeable glutamate channels, which are composed of NMDARs and AMPA receptors (AMPARs) (Steinhäuser and Gallo, 1996). One study showed that $\mathrm{Ca}^{2+}$ enters mature hippocampal astrocytes mainly through NMDARs. Extracellular $\mathrm{Ca}^{2+}$ can also enter astrocytes through voltage-gated calcium channels (VGCCs), and the expression of VGCCs seems to decrease with age (Steinhäuser and Gallo, 1996). The activation of transient receptor potential C-type channels (TRPCs) can also induce the entry of extracellular $\mathrm{Ca}^{2+}$ into astrocytes. As a non-selective calcium-permeable cation channel, TRPC can not only be activated by the production of IP3 and depletion of stored $\mathrm{Ca}^{2+}$ (Birnbaumer, 2009), but also participates in the increase of intracellular $\mathrm{Ca}^{2+}$ through store-operated $\mathrm{Ca}^{2+}$ entry independent of the PLC/IP3 signal cascade (Schwarz et al., 2019).

It is certain that the $\mathrm{Ca}^{2+}$ signal can regulate the release of glial transmitters in astrocytes. However, there must be other determinants of their release, such as the subcellular localization of receptors relative to internal calcium storage.

As mentioned earlier, $\mathrm{Ca}^{2+}$ is closely involved in the interconnection between neurons and astrocytes. Astrocytes can affect neuronal excitability, neurovascular coupling, synaptic electrophysiology, and synaptic plasticity by regulating $\left[\mathrm{Ca}^{2+}\right]_{\mathrm{i}}$. The effects of these signals on synaptic plasticity are not explained in detail here. At present, it is believed that $\mathrm{Ca}^{2+}$ has a prominent remodeling effect on neurons mainly by affecting the release of glial transmitters from astrocytes. According to previous studies, different receptor agonists such as glutamate and GABA can increase $\left[\mathrm{Ca}^{2+}\right]_{\mathrm{i}}$ in astrocytes and induce astrocytes to release glial transmitters, including glutamate, GABA, D-serine (D-ser), ATP, and prostaglandins. The relationship between $\mathrm{Ca}^{2+}$ and glial transmitter release is summarized in Figure 1. The release of GABA, glutamate, and ATP can increase $\left[\mathrm{Ca}^{2+}\right]_{\mathrm{i}}$ by activating presynaptic receptors, whereby ATP mainly activates presynaptic $\mathrm{P} 2 \mathrm{Y} 1$ receptors from the $\mathrm{P} 2$ receptor family, while glutamate induces NMDAR-mediated currents to regulate the release of synaptic vesicles to affect synaptic strength and regulate synaptic plasticity (Fattorini et al., 2019). In addition, $\mathrm{Ca}^{2+}$ waves also mediate the synaptic plasticity of toxic acetylcholine receptors in the somatosensory cortex and play a role in synaptic structural integrity, which depends on IP3R2 signals and extracellular D-ser (Norio et al., 2011). In a mouse model lacking the IP3 signal, the coverage of astrocytes in asymmetric synapses decreased and led to changes in the release of glutamatergic transmitters (Mariotti et al., 2015). In conclusion, the $[\mathrm{Ca} 2+]$ i transient $\left[\mathrm{Ca}^{2+}\right]_{i}$ of astrocytes is essential both for the regulation of neuronal activity and synaptic plasticity.

\section{ROLES OF ASTROCYTIC FACTORS IN THE REGULATION OF EXCITATORY SYNAPTIC FUNCTION}

\section{Astrocytic1 Neurotransmitters}

Synaptic plasticity has many manifestations. The typical types of synaptic plasticity include LTP and LTD. Studies have shown that astrocytes can regulate synaptic transmission and participate in the regulation of excitatory synaptic structure and function by releasing glial transmitters such as glutamate and D-ser in response to $\mathrm{Ca}^{2+}$ influx (Araque et al., 2014).

\section{Glutamate}

There are multiple glutamate release mechanisms in astrocytes. Under physiological conditions, glutamate is mainly released from astrocytes by exocytosis. Other $\mathrm{Ca}^{2+}$-release channels, such as $\mathrm{Ca}^{2+}$-dependent semi-channels, anion transporter channels, and $\mathrm{P} 2 \mathrm{X}$ receptor channels are mostly closed under physiological conditions, and are mainly related to pathological states such as epilepsy and injury (Vardjan et al., 2017).

As mentioned earlier, the neurotransmitters and other signals released by the synapses of neurons increase the level of intracellular $\mathrm{Ca}^{2+}$, which in turn activates exocytosis of glutamate by astrocytes and also activates glutamate receptors on the synaptic membrane. For example, glutamate exocytosis can activate presynaptic NMDA receptors (NMDARs) to increase $\mathrm{Ca}^{2+}$ levels and the excitatory current, thereby enhancing excitatory synaptic transmission in hippocampal dentate granule cells (Jourdain et al., 2007). In other words, this type of LTP is caused by the activation of NMDARs. In addition, NMDAR-dependent LTD or LTP can also be affected by the increase or decrease of AMPAR abundance in the postsynaptic membrane.

The activation of group I metabotropic glutamate receptors is reflected in the decrease of postsynaptic AMPAR abundance, 


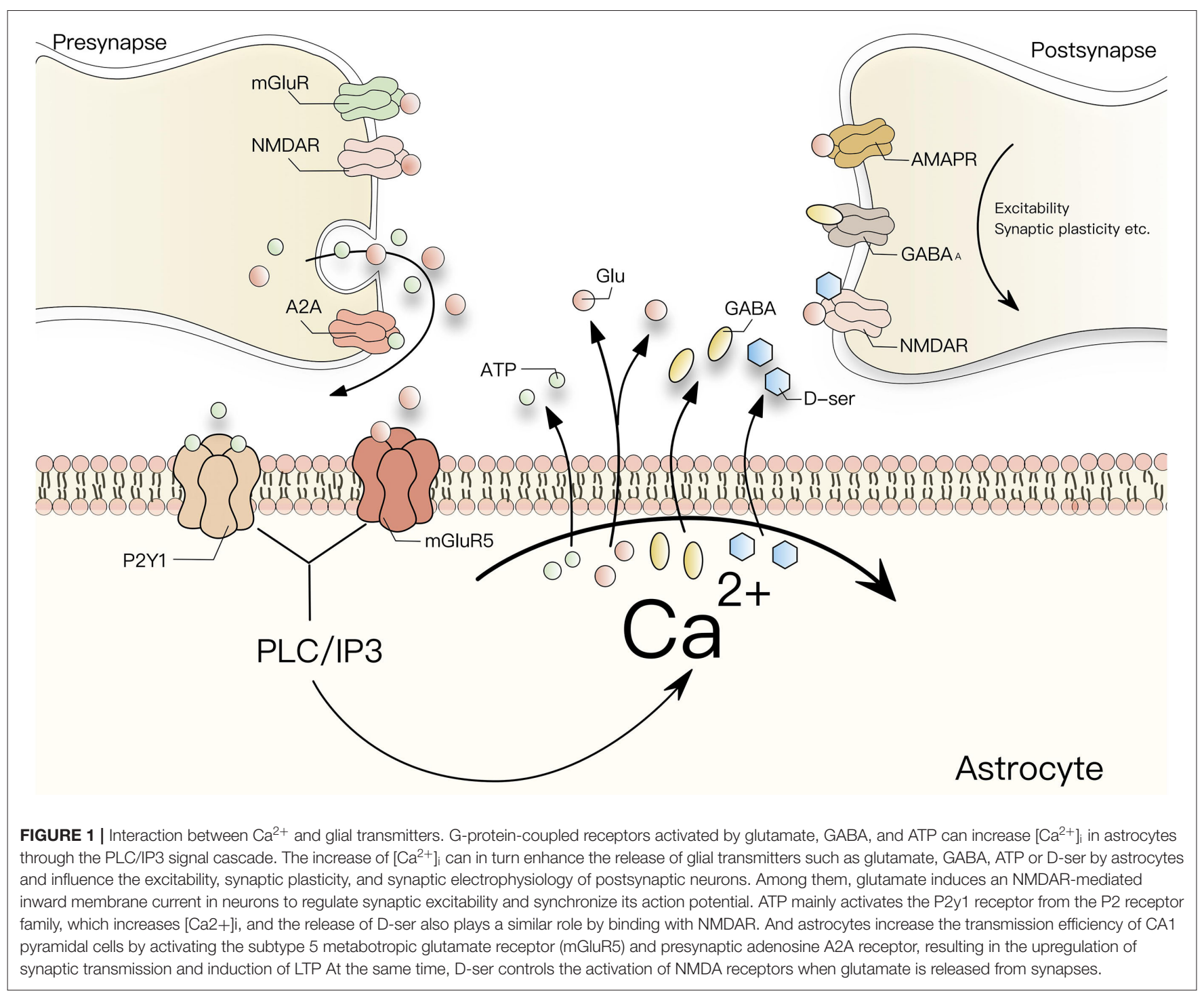

while LTD is induced when the number of AMPAR increases (Skowrońska et al., 2019). Although the number of postsynaptic AMPAR is always changing, synaptic transport through AMPAR can achieve dynamic balance, which is a complex process involving extracellular signaling circulation, and lateral diffusion (Choquet, 2018). More specifically, AMPAR is mobility as synaptic receptors, and both for extracellular circulation and lateral diffusion, reveal a continuous high mobility exchange of AMPAR between synaptic and extrasynaptic membranes.The extrasynaptic membrane of hippocampal pyramidal neurons contains a large number of AMPARs, which can be used as reserve materials, and dynamically exchange between synaptic and extra-synaptic sites through lateral diffusion (Tao-Cheng et al., 2011). Also, AMPAR can circulate between the intracellular pool and the cell surface pool through exocytosis or endocytosis, and through extracellular circulation to control its dynamic balance. This balance can be changed according to the needs of neuronal activity (Opazo and Choquet, 2011). For example, in the process of LTP, AMPAR can increase the abundance of postsynaptic AMPAR through lateral diffusion, and correspondingly, AMPAR excreted to maintain the dynamic balance naturally acts as an extra-synaptic reservoir (Makino and Malinow, 2009; Penn et al., 2017). Conversely, during LTD, the endocytosis of AMPAR reduces its activity from the postsynaptic membrane to reduce synaptic activity. In addition, the release of glutamate can trigger another form of LTP that does not depend on postsynaptic NMDAR-mediated signals but requires presynaptic mGluR activation (Perea and Araque, 2007).

As the third subfamily of iGluRs, the kainate receptors (KARs) have received much less attention. There are five members in the KARs family, GluK1 to GluK5, all with different functions. GluK2 and GluK5 are mainly found in neurons. GluK3 is expressed in granule cells of the dentate gyrus, while GluK1 is widely expressed during the entire whole process of growth and development but is confined to hippocampal intermediate neurons after full development (Lerma et al., 2001; 
Pinheiro et al., 2007; Vesikansa et al., 2007). In contrast to AMPARs, KAR-mediated currents are small in amplitude with slow activation and deactivation kinetics (Castillo et al., 1997; Frerking et al., 1998; Vignes et al., 1998). KARs are slowly activated or inactivated by Neto protein, an auxiliary subunit of KARs (Lerma, 2011; Jon et al., 2016) which gives KARs a variety of abilities to regulate synaptic plasticity (Straub et al., 2011; Sylwestrak and Ghosh, 2012; Lerma and Marques, 2013). Just like other iGluRs, KARs can be activated by Go protein, phospholipase C (PLC) and protein kinase C (PKC) metabolic cascade, but the mechanism of KAR activation in this atypical metabolic pathway has not been well-explained (Dubois et al., 2016; Duan et al., 2018; Tao et al., 2019). KARs are widely distributed in the presynaptic area (Schmitz et al., 2001; Lerma, 2006), and, together with multiplicity of signaling mechanisms of KARs, this may be a powerful factor affecting the regulation of presynaptic plasticity.

In addition to the iGluRs, the effects of astrocytic glutamate on mGluRs are also significant for the regulation of synaptic plasticity. In fact, mGluRs are extensively involved in the regulation of neuronal excitability and synaptic transmission throughout the CNS, as glutamate can regulate neuronal cell excitability as well as synaptic transmission through the second messenger signaling pathway (Sladeczek et al., 1985; Sugiyama et al., 1987). Metabotropic glutamate receptors (mGluRs) are differentially expressed in various cell types throughout the CNS, and to date, eight subtypes of mGluRs (Sladeczek et al., 1985; Sugiyama et al., 1987) have been characterized and classified into three subgroups based on sequence homology and cellular signaling activation (Gerber et al., 2007). The first group includes mGluR1 and mGluR5, the second group includes mGluR2 and mGluR3, while the third group includes mGluR4,6,7,8. The mGluRs are widely distributed in neurons, except for mGluR4, which is mainly distributed in the retina (Table 1).

The iGluRs and the mGluRs are constantly regulated in both directions, and group I mGluR activators enhance the activation of NMDAR-containing GluN2A/2B within minutes (Fitzjohna et al., 1996; Lan et al., 2001; Benquet et al., 2002). Interestingly, this effect appears to be a cell context specific, as enhancement of NMDAR occurs primarily in hippocampal neurons and NMDAR is inhibited in cortical neurons. Similar findings were reported for group I mGluR activation leading to the enhancement in CA1 neurons and inhibition of NMDAR currents in CA3 neurons (Grishin, 2004).

Group I mGluRs can also regulate other iGluRs. Internalization of receptors as a classical form of regulation leads to a reduction in the abundance of membrane receptors and downregulation of their function. In the context of studies on synaptic plasticity, the main focus has been on AMPAR, and group I mGluRs can lead to their internalization through a variety of kinases and phosphatases (Moult, 2006). In fact, not only AMPAR is affected by mGluR-induced internalization of iGluRs, since the number of NMDARs also decreases in response to mGluR activation (Snyder et al., 2001). From the aspect of kainic acid, mGluR in group I mainly enhance
TABLE 1 | Distribution of mGluR in CNS.

\begin{tabular}{|c|c|c|c|}
\hline Group & Receptor & CNS distribution & References \\
\hline \multirow[t]{3}{*}{ Group I } & mGluR1 & $\begin{array}{l}\text { Widespread in neurons } \\
\text { (cerebellar olfactory tubercle, } \\
\text { cerebral cortex, dentate gyrus, } \\
\text { lateral septal nucleus, striatum, } \\
\text { nucleus accumbens, amygdaloid } \\
\text { nuclei, substantia nigra pars } \\
\text { reticulata and cerebellar cortex) }\end{array}$ & $\begin{array}{l}\text { Pin and Duvoisin, } \\
\text { 1995; Kniazeff } \\
\text { et al., 2011; } \\
\text { Ribeiro et al., } 2011\end{array}$ \\
\hline & mGluR5 & $\begin{array}{l}\text { Widespread in neurons } \\
\text { (cerebral,cortex,Hippocampus, } \\
\text { subiculum,olfactory bulb,striatum } \\
\text { nucleus accumbens lateral } \\
\text { septal nucleus) }\end{array}$ & $\begin{array}{l}\text { Abe et al., 1992; } \\
\text { Shigemoto et al., } \\
\text { 1993; Romano } \\
\text { et al., } 1995\end{array}$ \\
\hline & & Astrocytes & $\begin{array}{l}\text { Biber et al., 2010; } \\
\text { Schools and } \\
\text { Kimelberg, } 2015\end{array}$ \\
\hline \multirow[t]{3}{*}{ Group II } & mGluR2 & $\begin{array}{l}\text { Widespread in neurons } \\
\text { (cerebellar cortex and olfactory } \\
\text { bulb) }\end{array}$ & $\begin{array}{l}\text { Ohishi et al., 1993, } \\
1994\end{array}$ \\
\hline & mGluR3 & $\begin{array}{l}\text { Widespread in neurons } \\
\text { (cerebellar olfactory tubercle, } \\
\text { cerebral cortex, dentate gyrus, } \\
\text { lateral septal nucleus, striatum, } \\
\text { nucleus accumbens, amygdaloid } \\
\text { nuclei, substantia nigra pars } \\
\text { reticulata and cerebellar cortex) }\end{array}$ & $\begin{array}{l}\text { Tanabe et al., } \\
\text { 1993; Testa et al., } \\
\text { 1994; Petralia } \\
\text { et al., 1996; Ohishi } \\
\text { et al., } 2010\end{array}$ \\
\hline & & Astrocyte & $\begin{array}{l}\text { Tanabe et al., } \\
\text { 1993; Ohishi et al., } \\
\text { 1994, } 2010\end{array}$ \\
\hline \multirow[t]{4}{*}{ Group III } & mGluR4 & most intense in the cerebellum & $\begin{array}{l}\text { Fotuhi et al., 1994; } \\
\text { Kinoshita et al., } \\
\text { 1996; Makoff } \\
\text { et al., 1996; Azkue } \\
\text { et al., } 2015\end{array}$ \\
\hline & mGluR6 & Retina & $\begin{array}{l}\text { Nakajima et al., } \\
1993\end{array}$ \\
\hline & mGluR7 & Widespread in neurons & $\begin{array}{l}\text { Kinoshita et al., } \\
2015\end{array}$ \\
\hline & mGluR8 & $\begin{array}{l}\text { Lower and more restricted than } \\
\text { mGluR } 4 / 7\end{array}$ & $\begin{array}{l}\text { Duvoisin et al., } \\
\text { 1995; Saugstad } \\
\text { et al., 1997; Corti } \\
\text { et al., } 1998\end{array}$ \\
\hline
\end{tabular}

containing GluK5 through PKC-mediated phosphorylation (Rojas et al., 2013).

Regrettably, there are only a few studies focusing on the regulation of iGluRs by group II and group III. For example, agonists of mGluR2 and mGluR3 can enhance NMDARs through a kinase-mediated pathway (Rosenberg et al., 2016). In the regulation of synaptic plasticity, iGluRs and mGluRs exhibit extensive antagonism and synergy. As mentioned above, group I mGluR mediates the enhancement of NMDAR, and the activation of group I mGluRs help increase the LTP amplitude in CA1 and CA3, whereby (the increase of LTP amplitude in CA1 is mainly influenced by mGluR5, and that in CA3 is influenced by mGluR1 (Berretta et al., 1993; Daniel et al., 1994; Lu et al., 
1997). In addition, mGluR5 (Table 1), which is widely distributed in astrocytes, can not only promote the release of glutamate and apoptosis of astrocytes by activating and inducing IpA formation and intracellular $\mathrm{Ca}^{2+}$ increase (Miller et al., 1995; Pasti et al., 1997), but also inhibit microglia-related neuroinflammation by stimulating the MAPK pathway and PLD signaling (Servitja et al., 2001; Peavy and Conn, 2010).

The enhancement of mGluR-LTD in the hippocampus is strongly related to FXS, which will be described in detail in the disease-related part later. The mGluR-LTD depends on the increase of intracellular $\mathrm{Ca}^{2+}$ and the activation of postsynaptic group I mGluRs, especially the mGluR1 receptor (Alba et al., 1994; Shigemoto et al., 1994), rather than NMDAR. This is a key feature of mGluR-mediated LTD. However, NMDAR-LTD and mGluR-LTD are not mutually exclusive, and the induction mechanisms of these two LTD are inconsistent. Nevertheless, LTD in the perinasal cortex and amygdala requires the synergy of NMDAR and mGluRs for activation (Wang and Gean, 1999; Cho et al., 2000).

\section{D-Serine}

As mentioned above, NMDARs play an important role in excitatory nerve transmission and synaptic plasticity. However, the activation of NMDARs does not depend solely on glutamate, and requires D-ser or glycine as a co-agonist to bind to the GluN1 and GluN2 subunits, respectively. D-ser has also been thought to mediate some aspects of NMDAR-dependent neurodegeneration as the main NMDAR co-agonist in the forebrain (Billard, 2012).

As a typical glial transmitter, D-ser is synthesized by serine racemase (SR), which converts L-serine into D-serine. Selective deletion of SR can impair NMDAR-dependent synaptic plasticity (Benneyworth et al., 2012; Perez et al., 2017). Astrocytes supply the L-serine necessary for SR activity to neurons, which convert it into D-ser (Wolosker and Radzishevsky, 2013). Then, the D-ser synthesized by the neuron will be released to activate NMDAR (Hagit et al., 2016). Accordingly, the supply of L-serine by astrocytes promotes the synthesis of $\mathrm{D}$-ser in neurons. As a co-agonist of NMDAR, D-ser then participates in the regulation of synaptic plasticity.

$\mathrm{Ca}^{2+}$-dependent $\mathrm{D}$-ser release is also involved in the regulation of synaptic plasticity. Repeated synaptic activity increases the free $\mathrm{Ca}^{2+}$ of astrocytes and increases the production of $\mathrm{D}$-ser in astrocytes in a short period of time. $\mathrm{Ca}^{2+}$-dependent $\mathrm{D}$-ser release induces NMDAR-dependent LTP on excitatory synapses and controls the plasticity of thousands of nearby NMDAR-dependent excitatory synapses (Henneberger et al., 2010; Zhuang et al., 2011). Besides, the H1 receptor in the hippocampal CA1 region promotes the activity of NMDAR and induces the enhancement of EPSC and LTP in the synapses of neurons depending on the continuous activation of astrocytes and release of D-serine (Masuoka et al., 2019).

\section{ATP}

ATP can provide energy for cells as a direct energy material in vivo, and it is also one of the main diffusion signal molecules released by astrocytes. The ATP response in rodents is mediated by two P2 receptor families. The P2X receptor is a ligandgated cationic channel that is permeable for $\mathrm{Na}^{+}, \mathrm{K}^{+}$, and $\mathrm{Ca}^{2+}$ (Burnstock and Kennedy, 2011; Saez-Orellana et al., 2015). ATP is the main endogenous agonist of all $\mathrm{P} 2 \mathrm{X}$ receptors. $\mathrm{P} 2 \mathrm{Y}$ receptors are G-protein-coupled receptors, which activate $\mathrm{Ca}^{2+}$ release by stimulating phospholipase C (PLC) (Mohapatra et al., 2011). The $\mathrm{Ca}^{2+}$ signal of human fetal astrocytes induced by ATP is completely mediated by $\mathrm{P} 2 \mathrm{Y} 1$ and $\mathrm{P} 2 \mathrm{Y} 2$ receptors, while $\mathrm{P} 2 \mathrm{X}$ receptors do not affect it (Muller and Taylor, 2017; Lalo et al., 2019). When ATP is converted into adenosine, it can interact with A1 and A2A receptors to inhibit or enhance excitatory synaptic transmission. In the process of synaptic transmission, astrocytes increase the transmission efficiency of CA1 pyramidal cells by activating the subtype 5 metabotropic glutamate receptor (mGluR5) and presynaptic adenosine A2A receptor, resulting in the upregulation of synaptic transmission and induction of LTP (Panatier et al., 2011). The types of receptors activated by ATP affect different functions of astrocytes. For example, local $\mathrm{Ca}^{2+}$ responses are mediated by mGluR5, while other types of glutamate receptors such asNMDA receptors are involved in other forms of astrocyte excitation and excitatory synaptic plasticity (Halassa and Haydon, 2010).

\section{Extracellular Matrix}

ECM molecules derived from astrocytes are also important factors in regulating synaptic function. In the central nervous system, the most prominent extracellular matrix structure is the peripheral neural network (PNN) (Lau et al., 2013). The PNN has the functions of producing polyanions that buffer the local environment, as well as capturing and locally concentrating growth factors and nutrients. Additionally, it can act as a diffusion barrier for extracellular signal molecules, including neurotransmitters in synapses, which helps prevent neurotransmitters from overflowing. Accordingly the PNN plays a role in stabilizing synapses, synaptic plasticity, and neuronal surface isolation (Suttkus et al., 2016; Sonntag et al., 2018).

Structurally, the PNN maintains the stability of chondroitin sulfate proteoglycans (CSPGs) binding to the hyaluronic acid (HA) skeleton by connecting proteins (Crtl1/Hapln1 and Bral2/Hapln4). In terms of composition, $\mathrm{HA}$ is the main component of the PNN, and CSPGs are its main proteoglycans. In the central nervous system, many CSGPs with different core proteins are secreted by astrocytes, such as brevican, versican and so on (Bandtlow and Zimmermann, 2000; John et al., 2006).

In EMC, the PNN is highly dynamic. After maturation, the PNN will establish a microenvironment between neurons and synapses, which will affect synaptic plasticity and determine where synapses form. The loosening of the PNN can destroy the stability of the inhibitory environment and release growth factors to promote synaptic plasticity (Dzyubenko et al., 2016).

When chondroitinase is injected into the central nervous system, it damages the PNN-like structure by destroying the chondroitin sulfate chain and hyaluronic acid (HA), which in turn impairs the transmission of excitatory synapses (Pyka et al., 2011). Therefore, PNN is a powerful factor in the effect of astrocytes on synaptic plasticity. 


\section{Astrocyte Surface Proteins in Direct Contact With Synapses}

Synapses are also affected by the direct adhesion between neurons and astrocytes. One factor mediating astrocyte-neuron adhesion is $\gamma$-Pcdh, and this astrocyte protein can promote the development of excitatory and inhibitory synapses (Garrett and Weiner, 2009; Pyka et al., 2011).

Studies have shown that the activation of protease-activated receptor 1 (PAR1) in hippocampal CA1 astrocytes leads to the opening of the $\mathrm{Ca}^{2+}$-dependent glutamate-permeable anion channel Best1, which mediates $\mathrm{Ca}^{2+}$-dependent glutamate release from astrocytes, thereby increasing the concentration of glutamate in the synaptic space (Woo et al., 2012). Synaptic NMDAR is the main target of Best1-mediated glutamate release in astrocytes. Increased activation of synaptic NMDAR leads to the enhancement of NMDAR-dependent synaptic transmission. When synaptic glutamate increases glutamate secretion in astrocytes mediated by Best1, the threshold of NMDARdependent LTP decreases (Park et al., 2015).

\section{Slow-Acting Astrocyte-Derived Cytokines}

Astrocytes can secrete a variety of cytokines and neurotransmitters that participate in the regulation of synaptic plasticity. Hevin is a synaptic protein secreted by astrocytes. In the developing cortex, Hevin is necessary for maintaining synaptogenesis and maturation of the dendritic structure in the thalamic cortex (Risher et al., 2014). The transsynaptic adhesion between presynaptic neurexins (NRX) and postsynaptic neuroligins (NL) is essential for the formation and maturation of excitatory and inhibitory synapses (Baudouin and Scheiffele, 2010). Hevin can bridge Neuresin- $1 \alpha$ and NeuroLigin-1B to assemble glutamatergic synapses. Thus, astrocytes change the cross-synaptic interaction between NLS and NRXs via Hevin, thereby regulating the formation and plasticity of excitatory synapses (Singh et al., 2016). Other synaptic proteins such as thrombospondin (TSP) play an important role in the formation of excitatory synapses (Chung et al., 2015; Baldwin and Eroglu, 2017). During development, the nervous system undergoes a process of forming a large number of neural circuits, during which the expression of TSP-1 and TSP-2 in astrocytes is maintained at a high level. Conversely, a lack of TSP will lead to a decrease in the number of excitatory synapses (Christopher et al., 2018). Glypicans can also increase the formation of excitatory synapses. Glypican 4 and Glypican6 secreted by astrocytes can recruit AMPA glutamate receptors to the surface of postsynaptic cells and induce the formation of active excitatory synapses by increasing the density of receptors on the surface of postsynaptic cells (Allen et al., 2012; Baldwin and Eroglu, 2017; FarhyTselnicker et al., 2017). Transforming growth factor $\beta 1$ (TGF- $\beta 1$ ) is also secreted by astrocytes. As a member of the TGF- $\beta$ superfamily, TGF- $\beta 1$ has been recognized as a neuroprotective and neurotrophic factor in previous studies. Recent studies on the role of TGF- $\beta 1$ in synaptic transmission have found that it is expressed in hippocampal neurons in an activity-dependent manner and participates in synaptic regulation (Caraci et al., 2015, 2018). In vivo, the levels of AMPA and NMDA receptor subunits increase in the hippocampi of mice with TGF- $\beta 1$ overexpression, and the induction of excitatory synapses by TGF- $\beta 1$ is mediated by NMDA receptor activity and the NMDA coactivator D-serine (Bae et al., 2011).

In addition, TGF- $\beta 1$ can also induce a switch from the early stage of LTP, which is independent of protein synthesis (E-LTP) into the late stage that is dependent on protein synthesis (L-LTP), which indicates that TGF- $\beta 1$ can increase neuronal excitability by inducing long-term dissimilation of ganglia (Caraci et al., 2015).

Brain-derived neurotrophic factor (BDNF) also plays an important role in regulating synaptic plasticity. BDNF works through two receptor systems, TrkB (tropomyosin-related kinase B) and P75NTR (Baldwin and Eroglu, 2017; Holt et al., 2019). Interestingly, the interaction of BDNF through p75NTR and Trk receptors often has opposite effects. For example, Trk receptor attachment almost always promotes neuronal survival and differentiation (Patel et al., 2000), whereas p75NTR involvement often promotes apoptosis (Defreitas et al., 2001). Similarly, in the case of synaptic plasticity, BDNFmediated activation of TrkB promotes hippocampal LTP, whereas the interaction of neurotrophic factor with p75NTR instead promotes hippocampal LTD. A major pathway involved in p75NTR activation by neurotrophic factors is the Jun kinase signaling cascade, which leads to the activation of p53 and apoptosis. In addition, the Jun kinase cascade induces the expression of Fas ligands in neuronal cells, which promotes apoptosis by activating Fas receptors. There are important intermediates in the Jun kinase cascade pathway, such as NRIF and the E3 ubiquitin ligase Traf6. When these intermediates are absent, Jun kinase signaling in sympathetic neurons will also be blocked (Yeiser, 2004; Linggi et al., 2005). In PC12 cells and sympathetic neurons, the activation of the Jun kinase cascade involves $\mathrm{Cdc42}$, and apoptosis is strongly inhibited by dominant-negative Cdc42 mutations (Bazenet and Rubin, 1998). Binding of neurotrophic factor to p75NTR also promotes NF$\kappa \mathrm{B}$ activation, thereby promoting NF- $\kappa \mathrm{B}$-dependent neuronal survival (Middleton, 2000). The involvement of neurotrophic factors in p75NTR also controls Rho family GTPase activity. It was shown that p75NTR activates RhoA through direct interaction, thereby inhibiting neurite growth. The binding of neurotrophic factor to p75NTR eliminates p75NTR-dependent RhoA activation and stimulates neurite growth (Yamashita et al., 1999). All of these studies illustrate the self-evident importance of p75NTR as a death receptor in neural and synaptic development.

In terms of synaptic plasticity, the effect on LTP is mainly mediated by the TrkB receptor. Stable LTP in rodent DG requires a BDNF-TrkB signal period lasting more than $8 \mathrm{~h}$ after LTP induction (Panja et al., 2014). Unprocessed BDNF is also called proBDNF, and its synaptic effect is opposite to that of mature BDNF, which acts through TrkB. By contrast, proBDNF has a high affinity for the p75NTR (Chao and Bothwell, 2002; Lu, 2003). ProBDNF binds to P75NTR first, and their binding induces LTD (Rösch et al., 2005). In the normal physiological state, the expression of P75NTR in the nervous system is very limited, and it is upregulated in pathological conditions such as inflammation or seizures. Therefore, the influence of BDNF on LTD is minimal (Figure 2). Studies have also shown that 
cholecystokinin (CCK) and glutamate cooperate with the CCK receptor and mGluR5 in astrocytes to trigger the release of the glial transmitter ATP, which increases the release of GABA from adjacent inhibitory terminal synapses, and switches the state of GABAergic synapses in the dorsomedial hypothalamic nucleus of rats from LTD to LTP (Crosby et al., 2018).

\section{ROLES OF ASTROCYTIC FACTORS IN THE REGULATION OF INHIBITORY SYNAPTIC FUNCTION}

\section{Astrocytic Exocrine1 Factors}

GABA is the main inhibitory neurotransmitter, and astrocytes express the GABA transporters GAT1 and GAT3 (Scimemi, 2014). Changes in the expression and activity of GAT1 or GAT3 affect inhibitory synaptic transmission in inter-hippocampal neurons (Beenhakker and Huguenard, 2010). GATs control the excitability of neurons in the neural network by regulating the level of extracellular GABA (Muthukumar et al., 2014). Furthermore, there is a signaling pathway between astrocytes and the GABA system. Astrocytes can enhance the current mediated by GABA in neurons and increase the transmission of inhibitory synapses in hippocampal CA1 neurons through $\mathrm{Ca}^{2+}$ signals. GABA selectively opens the $\mathrm{Cl}$-channel by activating the type A GABA receptor (GABAAR), which induces an inhibitory potential and mediates rapid synaptic inhibition. The activation of GABAb elevates the $\mathrm{Ca} 2+$ level, which at the same time enhances the $\mathrm{Ca} 2+$-dependent release of gliotransmitters from astrocytes (Kang et al., 1998). It is now generally accepted that synaptic inhibition is mediated by GABAA receptors and that potentiation involves astrocyte $\mathrm{GABAB}$ receptors, astrocyte glutamate release, and presynaptic metabotropic glutamate receptors. And interneuron activity regulates the release of transmitters from the same synapses according to the firing rate, which suggesting that mechanisms of synaptic inhibition can coexist at the same synapse as those responsible for synaptic potentiation and that the end result is regulated by the firing rate of the interneuron (Perea et al., 2016). In a mouse model of Alzheimer's disease $(\mathrm{AD})$, the excess $\mathrm{GABA}$ released by reactive astrocytes through the Best1 channel in the hippocampus acts on presynaptic GABA receptors and leads to catatonic inhibition of the dentate gyrus granulosa cells in the hippocampus (Lee et al., 2010; Jo et al., 2014). In terms of memory impairment caused by $\mathrm{AD}$, inhibition of $\mathrm{GABA}$ produced by reactive astrocytes or blocking of GATs by different means can restore synaptic plasticity, learning, and memory impairment caused by $\mathrm{AD}$. The application of GABA receptor antagonists in AD mice can improve hippocampal LTP, alleviate the learning impairment, and protect memory to different degrees (Yuji et al., 2008). Therefore, it stands to reason that inhibition of the synthesis or release of GABA may be a powerful method for treating memory impairment caused by $\mathrm{AD}$.

TGF- $\beta 1$ not only promotes the formation of excitatory protrusions, but also promotes the formation of inhibitory synapses. It induces the formation of inhibitory synapses by increasing the phosphorylation of CaMKII downstream of
NMDA receptors, as well as increasing the expression of NL2 and aggregation of Gephyrin/NL 2, which is an important component of inhibitory synapses (Diniz et al., 2015).

At present, it is believed that Chordin-like1 (Chrdl1) factor secreted by astrocytes can regulate the level of synaptic GluA2AMPAR, mainly by increasing its quantity. In the developing brain, an important sign of the stability and maturation of excitatory glutamatergic synapses is that AMPAR subtypes on excitatory synapses are replaced by $\mathrm{Ca}^{2+}$-opaque channels containing GluA2 from $\mathrm{Ca}^{2+}$ permeable channels (Brill and Huguenard, 2008). In other words, by reducing the entry of calcium into postsynaptic cells, excitatory glutamatergic synapses tend to mature, stabilize, and limit synaptic plasticity mediated by $\mathrm{Ca}^{2+}$-dependent pathways (Henley and Wilkinson, 2016). However, the mechanism of the iconic transformation of stable mature synaptic AMPAR is not clear. Studies show that synaptic GluA2 AMPAR decreases and synaptic remodeling increases in response to changing sensory inputs in Chrdl1KO mice. In other words, Chrdl1 expressed by astrocytes limits synaptic plasticity by promoting GluA2-dependent synaptic maturation (Henley and Wilkinson, 2016).

With regard to the effect of ATP on LTD, researchers found that selective stimulation of astrocytes through ChR2 could lead to LTD, while blocking P2Y receptors in neighboring hippocampal neurons could prevent hLTD. Thus, ATP secreted by astrocytes mediates hippocampal LTD by activating neuronal P2Y1 receptors (Chen et al., 2013).

\section{Other Factors}

Recent studies have shown that the number and transmission of inhibitory synapses in neurons co-cultured with astrocytes is increased compared with neurons cultured alone, and their plasticity is enhanced (Sorg et al., 2016). Interestingly, these effects disappeared after treatment with selective Krebs cycle inhibitors such as fluoroacetic acid, indicating that the regulation of GABAergic synaptic development depends on a series of metabolic pathways in astrocytes, including the Krebs cycle. In other words, key metabolic enzymes expressed by astrocytes, such as glutamine synthetase, contribute to the plasticity of GABAergic neuronal networks (Kaczor and Mozrzymas, 2017).

\section{PRUNING EFFECT OF ASTROCYTES ON SYNAPTIC MORPHOLOGY}

In order to form functional neural circuits, the initially formed excess synapses are removed during brain development. This kind of pruning plays an important role in normal synaptic development and neural circuit formation in the CNS. Recent studies have found that astrocytes are involved in this synaptic pruning process.

\section{Direct Effects}

Synaptic phagocytic receptors MEGF10 and MerTK are expressed on the surface of astrocytes. Astrocytes can eliminate excitatory or inhibitory synapses by interacting with MEGF10 and MerTK, which can recognize phosphatidylserine in target fragments as an opsonic signal to initiate phagocytosis and 


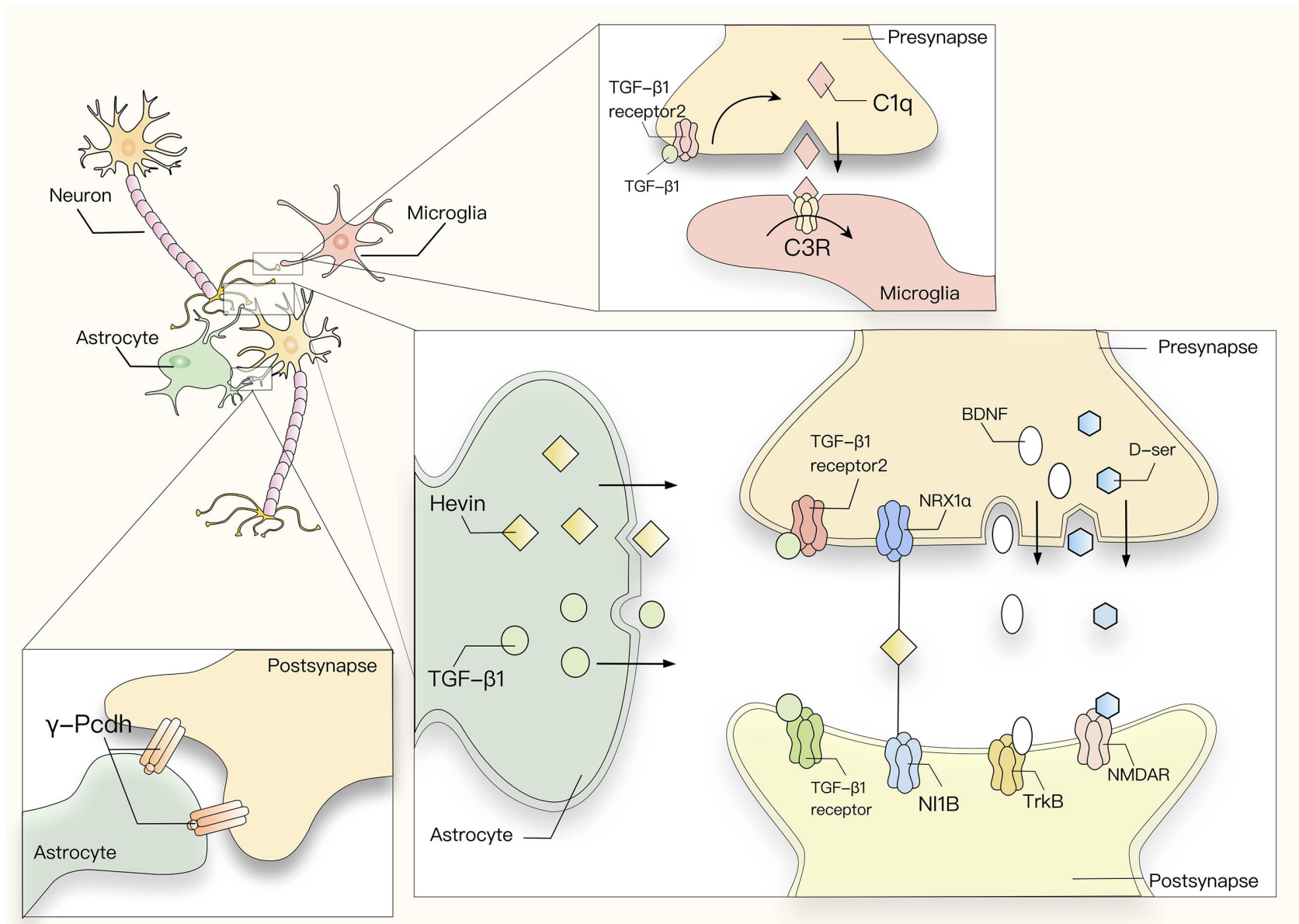

FIGURE 2 | Astrocytic cytokines and neurotransmitters participate in the regulation of synaptic plasticity. TGF- $\beta 1$ secreted by astrocytes induces the formation of inhibitory synapses by activating neuronal calcium/calmodulin dependent protein kinase II (CaMK II), or promotes the formation of excitatory synapses through an activity-dependent mechanism of the NMDA receptor together with its agonist D-ser. In addition, the development of both excitatory and inhibitory synapses is affected by astrocyte-neuron adhesion mediated by astrocytic $\gamma$-Pcdh. Astrocytes secrete Hevin to interact with two proteins, presynaptic NRX1 $\alpha$ and postsynaptic NL1b, which usually do not interact with each other, thus promoting synapse formation. On the other hand, BDNF regulates LTP mainly by binding to the TrkB receptor. Astrocytes can also induce the production of complement protein C1q by secreting TGF- $\beta$ and thereby acting on TGFBR2 in the synapse. C1q is recognized by microglia and triggers C3R-mediated phagocytosis.

drive synaptic remodeling (Figure 3) (Chung et al., 2013). This process of synaptic elimination is strongly dependent on neural activity. Recent studies have shown that astrocytes regulate synaptic elimination by activating purinergic signals in an ATP-dependent manner mediated by IP3R2 receptor release (Yang et al., 2016).

In addition, studies have shown that astrocytes can express Ephrin-B1, which is a membrane binding protein and the ligand of the $\mathrm{EphB}$ receptor. The cross-synaptic ephrin- $\mathrm{B} / \mathrm{EphB}$ interaction between neurons is essential for the formation and maintenance of synapses in the mouse brain (Henkemeyer et al., 2003; Grunwald et al., 2004; Xu and Henkemeyer, 2012). Astrocytic ephrin-B1 can compete with neuronal ephrin-B1 and trigger astrocyte-mediated elimination of synapses containing EphB receptors through cross-synaptic endocytosis. The loss of the neuronal EphB receptor weakens the ability of astrocytes expressing functional Ephrin-B1 to phagocytize synaptosomes in vitro, while the overexpression of Ephrin-B1 in astrocytes impairs long-term contextual memory. Thus, astrocytic EphinB1 regulates long-term contextual memory by limiting the formation of new synapses in the adult hippocampus (Koeppen et al., 2018).

Astrocytes are the main source of apolipoprotein $\mathrm{E}(\mathrm{ApoE})$ in the central nervous system and transport cholesterol to neurons through apolipoprotein $\mathrm{E}$ receptors, a member of the (LDLR) family of low-density lipoprotein receptors. ApoE exists in three subtypes, ApoE2, ApoE3, and ApoE4, whereby the latter is related to the decrease of dendritic spine density in memory retention (Caselli et al., 2004). The formation of dendritic spines is the most important feature of synaptic plasticity of mature neurons. At the same time, AopE4 is also the strongest genetic risk factor for late-onset Alzheimer's disease (LOAD). 


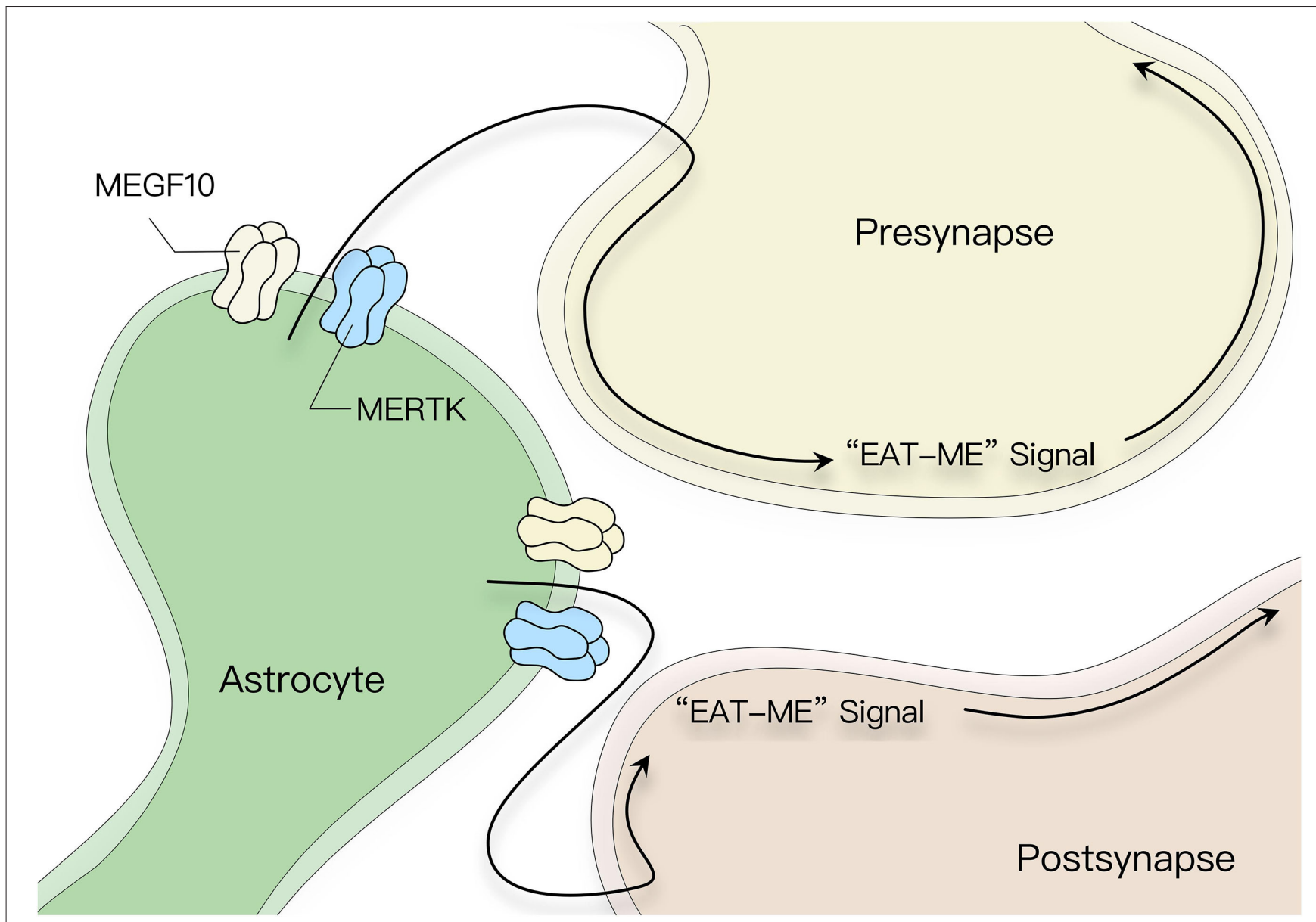

FIGURE 3 | Pruning effect of astrocytes on synaptic morphology. Astrocyte surface receptors MEGF10 and MerTK recognize phosphatidylserine in the target fragment as an opsonic signal, which induces astrocytes to eliminate superfluous synapses in the CNS.

\section{Indirect Effects}

Astrocytes can also initiate the classical complement cascade by secreting TGF- $\beta$ and inducing the expression of complement protein $\mathrm{C} 1 \mathrm{q}$ via the TGFBR2 receptor. C1q is then recognized and taken up by microglia through C3R-mediated phagocytosis (Schafer et al., 2012; Bialas and Stevens, 2013). In this way, astrocyte-derived TGF- $\beta$ regulates the expression of neuronal $\mathrm{C} 1 \mathrm{q}$ to initiate complement activation and microglial-mediated synaptic pruning (Figure 2).

In addition to complement C1q, IL-33 expressed by astrocytes also drives microglial synaptic phagocytosis and limits the number of excitatory synapses (Nguyen et al., 2020). These studies have shown that astrocytes can mediate the indirect effects of microglia-dependent synaptic elimination through $\mathrm{Clq}$ or IL-33.

\section{IMPLICATIONS OF ASTROCYTE CELL BIOLOGY FOR HUMAN DISEASES}

Astrocytes play important roles in a range of neurodevelopmental and psychiatric disorders in humans, and a better understanding of the function of astrocytes is needed to understand the pathological changes that lead to these disorders. Because astrocytes are so closely related to synapses and influence synapse formation, maturation and plasticity, their dysfunction may be closely associated with psychiatric disorders such as schizophrenia, autism and depression. In addition, astrocyte dysfunction has also been implicated in the pathology of human neurodevelopmental disorders such as Rett syndrome, fragile $\mathrm{X}$ syndrome, and Down syndrome.

\section{Astrocytes and Autism Spectrum Disorders} Autism spectrum disorders (ASD) are a heterogeneous group of neurological developmental conditions characterized by repetitive movements, impairments in social interaction, and altered vocal communication as the core symptoms (Lai et al., 2014). The exact pathophysiological basis of ASD is currently unknown, but a growing body of evidence supports dysregulated neuroinflammation and dynamic imbalances in synapse formation (Christopherson et al., 2005; Kucukdereli et al., 2011), pruning, elimination (Chung et al., 2013), and 
plasticity (Henneberger et al., 2010) as important factors contributing to ASD. Neuroinflammation is characterized by the persistent activity and proliferation of glial cells (e.g., astrocytes and microglia) following injury, infection, or disease. Astrocytes respond to neuroinflammation through morphological changes and the release of pro-inflammatory mediators (Hanisch and Kettenmann, 2007; Kierdorf and Prinz, 2013). Since astrocytes are closely associated with the connection and regulation of neural circuits, early disruption of astroglial homeostasis may lead to abnormal brain development and the ASD phenotype. Autopsy studies have found that individuals with ASD have increased numbers of neurons in the cerebral cortex, white matter, and especially the cerebellum (Morgan et al., 2010; Vargas et al., 2010). Western blot analysis indicated that GFAP protein expression was significantly increased in the cerebellum, frontal middle gyrus, and anterior cingulate gyrus of autistic patients, suggesting that astrocyte immunoreactivity was also increased (Vargas et al., 2010). Recent studies have shown that astrocytes control excitatory synaptogenesis through the secretion of platelet responsive protein (TSP), which acts through its neuronal receptor calcium channel subunit $\alpha 2 \delta-1$ (Eroglu et al., 2009). The $\alpha 2 \delta-1$ receptor is associated with neurological disorders such as epilepsy, neuropathic pain, intellectual disability and ASD (Newton et al., 2001; Iossifov et al., 2014). The small Rho GTPase Ras-Related C3 Botulinum toxin substrate 1 (RAC1) is a synaptic signaling protein downstream of the TSP- $\alpha 2 \delta-1$ axis. $\mathrm{RAC1}$ is a key component of the cascade that mediates synaptic and spinal growth (Christopher et al., 2018). This provides a new perspective for intervention in diseases including ASD and epilepsy, which are strongly associated with abnormal RAC1 signaling (Zeidán-Chuliá et al., 2013; Tejada-Simon, 2015). Since astrocytes can control neuronal RAC1 signaling through TSP$\alpha 2 \delta-1$, understanding the regulatory mechanism of the TSP$\alpha 2 \delta-1-R A C 1$ pathway in astrocytes may uncover new potential therapeutic targets for psychiatric disorders such as ASD in the future.

\section{Astrocytes and Rett Syndrome}

Rett syndrome (RTT) is a rare X-linked neurodevelopmental disorder resulting in a range of symptoms including autistic features, intellectual impairment, motor degeneration and autonomous nervous system abnormalities. Loss of function of the X-linked gene MECP2, which encodes methyl CpGbinding protein 2, is the main cause of RTT (Amir et al., 1999). MECP2 is both a transcriptional activator and a transcriptional repressor that regulates synaptic activity in an activity-dependent manner (Qiu et al., 2012). Although few early studies explored the role of glial cells in RTT, advances in technology have shown that MECP2 is expressed in both astrocytes and neurons (Grunseich et al., 2009). In fact, a recent study found that naïve dendrites of primary WT neurons co-cultured with MECP2deficient primary astrocytes were poorly developed compared to neurons co-cultured with WT primary astrocytes (Grunseich et al., 2009; Maezawa et al., 2009). Moreover, dendritic and synaptic morphology could be restored in vivo after restoring MECP2 (Lioy et al., 2011). These findings that MECP2-deficient astrocytes are detrimental to neuronal and dendritic growth and development, and that astrocytic MECP2 deficiency is an important cause of RTT. This morphological abnormality of neurons and dendrites has been attributed to neurotoxins released by MECP2-deficient astrocytes. However, how the astrocytes of RTT patients exert neurotoxic effects remains unknown. Furthermore, the transcription of the neurotrophic factor BDNF secreted by astrocytes is also regulated by MECP2, and overexpression of BDNF in MECP2-deficient mice improved motor function and restored the normal electrophysiological activity of somatosensory pyramidal neurons (Chang et al., 2006). Based on this, we can ask the question whether MECP2 can also control other factors secreted by astrocytes (such as TSPs, Hevin, etc.).

\section{Astrocytes and Fragile X Syndrome}

Among the many forms of inherited intellectual disability, Fragile X syndrome (FXS) is one of the most common. The trinucleotide repeat amplification of the Fragile $\mathrm{X}$ mental retardation 1 (FMR1) gene can lead to the loss of Fragile $\mathrm{X}$ mental retardation 1 protein (FMRP), which plays a number of important roles in the human body, such as binding to proteins involved in the regulation of genomic stability, cell differentiation and other processes (Contractor et al., 2015). It also plays an important role in the translation, transport and targeting of neuronal mRNAs, which in part control synaptic plasticity, development and elimination (Pfeiffer and Huber, 2009). FXS is mainly characterized by moderate to severe mental retardation, autistic traits, susceptibility to seizures, and behavioral abnormalities (Penagarikano, 2007). FMR1 knockout (KO) mice have phenotypes such as learning and intellectual deficits (Comery et al., 1997; Wisniewski et al., 2010), as well as changes of social behavior (Mckinney et al., 2010) that are similar to those of human FXS patients.

In vivo imaging studies on global FMR1 $\mathrm{KO}$ mice indicated that the lack of FMRP leads to reduced synaptic stability (Feng et al., 2010; Padmashri et al., 2011). In addition, the density of immature dendritic spines in cortical vertebral neurons is greatly increased in both FXS patients and adult FMR1 KO mice (Dailey and Smith, 1996). The morphology and density of dendritic spines has important implications for proper synaptic connectivity and function (Sala and Segal, 2014). Therefore, the lack of FMRP will lead to abnormal development of dendritic spines and thus affect synaptic connections and functions. For example patients with fragile $\mathrm{X}$ syndrome, the spine is usually longer and thinner than the average person and there are more spines exist per unit length of the dendrite (Irwin et al., 2001), which lead to a series of abnormalities social behavior such as verbal language deficits, reduced eye contact, social and generalized anxiety, sensory hypersensitivity, and difficulty regulating attention and activity levels (Lewis et al., 2010). These abnormalities in social behavior will continue from the onset into adulthood. Also, Patients with fragile X syndrome have a learning disability, as both learning and memory are impaired in patients with fragile $\mathrm{X}$ syndrome.. A recent study showed that selective deletion of the FMR1 gene in astrocytes results in reduced expression of the astroglial glutamate transporter protein GLT1 (Higashimori et al., 2016). The primary role of 
GLT1 is the regulation of the extracellular synaptic glutamate concentration, and GLT-1-mediated loss of glutamate uptake increases extracellular glutamate levels, thereby increasing excitability of layer $\mathrm{V}$ pyramidal neurons (Higashimori et al., 2016). The increased glutamate concentration may promote the growth of new dendritic spines (Kwon and Sabatini, 2011). Thus, the dysregulation of glutamate homeostasis caused by the absence of FMRP in astrocytes may partially explain the increased density of immature dendritic spines in FMR1 KO mice. Astrocytespecific deletion of FMR1 leads to a significant increase in immature spines in the motor cortex of mice, which manifests as abnormal synaptic morphology and reduced synaptic density in hippocampal neurons. This is due to the overproduction of dendrites during neuronal development and abnormal or insufficient pruning (Hodges et al., 2016). Interestingly, normal astrocytes prevent such abnormal synapses and reduced density of hippocampal neurons. Therefore, astrocytes play an important role in the abnormal synaptic development of FMR1 KO mice.

The group I mGluRs (mGluR1/5)-mediated LTD is a specific protein synthesis-dependent synaptic plasticity, which can be increased in FMR1KO mice (Huber et al., 2002). This groundbreaking discovery led to a new mGluR-based theory of FXS, which states that dysregulated mGluR1/5-mediated protein synthesis-dependent forms of synaptic plasticity contribute to the pathology of FXS (Bear et al., 2004). The synthesis of this specific protein leads to the internalization of AMPAR, which is the key mechanism of mGluR-LTD. Unfortunately, this mechanism seems to be damaged in FXS. Some studies have found that the mGluR5-mediated internalization of AMPAR increases when FMRP levels decrease (Nakamoto et al., 2007). Therefore, we have reason to believe that the role of FMRP in regulating mGluR1/5-induced protein synthesis may be the basis of LTD, exemplified in FXS mice. In addition, the sensitivity of mGluR1/5 to glutamate released by synapses in the hippocampus is enhanced in FXS mice, and the resulting loss of FMRP also leads to prolonged epileptiform discharges (Chuang, 2005; Bianchi et al., 2009). Moreover, the synthesis and release of endogenous cannabinoids in the hippocampus cannabinoid (eCB) mediated by mGluR1/5 is in enhanced following the deletion of FMRP (Zhang and Alger, 2010). In fact, there are also defects of the mGluR1/5-dependent pathway in other areas of the FXS mouse brain, such as abnormal synaptic plasticity of mGluR1/5-dependent neurons in the amygdala of FMR1 KO mouse (Suvrathan et al., 2010). Similar to the excessive LTD in the hippocampus, there is also excessive mGluR1/5-dependent LTD in the cerebellum of FXS mice (Koekkoek et al., 2005). Since different areas of the brain correspond to different functions, defects of the mGluR1/5-dependent pathway in different areas of the FXS brain may not only lead to cognitive dysfunction, but also lead to epilepsy (prolonged epileptiform discharges in the hippocampus), anxiety (abnormal synaptic plasticity in the amygdala) and motor deficits deficit (cerebellum). Although most of these studies are based on FXS animal models that may not always completely reflect the human phenotype, the successful validation of mGluR theory in different animal models further confirmed that FMRP plays a vital role in stimulationinduced mGluR1/5 signal transduction, and the potential value of glutamate derived from astrocytes via to activate mGluR1/5 as a therapeutic target for the treatment of FXS.

\section{Astrocytes and Down Syndrome}

Trisomy of chromosome 21 causes Down syndrome (DS), which is the most common cause of inherited mental retardation. Intellectual disability in DS includes deficits in cognitive function, interpersonal interaction and communication. Abnormalities in the structure and function of dendritic spines are one of the prominent features of DS. Altered dendritic spine morphology and reduced density may underlie altered neuronal and synaptic plasticity (Benavides-Piccione et al., 2004), ultimately leading to cognitive deficits. In the cortical and hippocampal structures of DS fetuses and neonates, the morphology of dendritic spines exhibits abnormal changes, such as unusually long spines, shorter spines, and a decrease in synaptic density (Marin-Padilla, 1972). It was found that this abnormal dendritic spine development as well as the reduced synaptic density and activity in DS patients is closely related to astrocytes, and that the astrocyte-secreted TSP-1 protein is a key factor that regulates the number and morphology of dendritic spines. TSP-1 levels are significantly reduced in the astrocytes of DS patients, and restoration of TSP-1 levels can rescue the aforementioned morphological abnormalities and synaptic defects in the dendritic structures of hippocampal and cortical neurons (Octavio et al., 2010). Such findings highlight the role of astrocytes in synaptic defects in DS, and although there are no effective methods to date to prevent or restore such synaptic defects, these results demonstrate the potential of astrocyte-secreted molecules such as TSP-1 as therapeutic targets for symptomatic relief in DS and other neurological disorders associated with synaptic defects.

Indeed, astrocytes may influence numerous neurological disorders in multiple ways, and it is well-known that targeting neurons will be far more difficult than targeting astrocytes, which speaks volumes about the potential of astrocytes as therapeutic targets for the prevention and treatment of related diseases.

\section{CONCLUSIONS}

The concept of a "tripartite synapse" allows us to re-examine the role of astrocytes in neural circuits, rather than simply treating them as supporting cells that passively fill the gaps between neurons. As a component of the synapse, astrocytes promote synaptogenesis and regulate synaptic connectivity by influencing synapse formation, elimination, and maturation by secreting proteins, lipids, and small molecules that bind to neuronal receptors. Synaptic dysfunction is a prominent feature of various neurological diseases, including Alzheimer's disease, autism, and schizophrenia. Therefore, the importance of astrocytes is selfevident. Astrocytes secrete neurotransmitters such as glutamate and ATP that affect intracellular $\mathrm{Ca}^{2+}$ levels by acting on corresponding metabolic receptors. Astrocytes also initiate and maintain $\mathrm{Ca}^{2+}$ waves between neurons and astrocytes, and induce LTP. We discussed that the increase of $\mathrm{Ca}^{2+}$ levels in astrocytes has a direct effect on the release of synaptic neurotransmitters and synaptic plasticity, but the research on the effects of astrocytic signal characteristics on the nervous 
system, such as the rate of $\mathrm{Ca}^{2+}$ increase in astrocytes, the rise in subcellular regions or the mechanism underlying the effects of IP3-dependent and -independent signals on the nervous system and astrocytes is still insufficient. At the same time, how the diverse glial transmitters are released from astrocytes under the influence of $\mathrm{Ca}^{2+}$, the release pathway, and the mechanism are still controversial. If future research can clarify these problems, it will greatly promote our understanding of how astrocytes affect synaptic plasticity.

In addition, most studies have focused on the role of astrocytes in regulating the formation, function or elimination of excitatory synapses, while little is known about the role of inhibitory synapses or circuits. Only a few studies have reported the mechanism through which astrocytes influence the development of GABAergic synapses and related neural circuits. At the same time, many studies have begun to explain the relationship between astrocytes and neurodegenerative diseases.

The connection of targeted neurons is much more difficult than that of targeted glial cells, so astrocytes are a better intervention target than neurons during growth and development. The study of the influence of astrocytes on synaptic plasticity in the development of neural circuits can

\section{REFERENCES}

Abe, T., Sugihara, H., Nawa, H., Shigemoto, R., Mizuno, N., and Nakanishi, S. (1992). Molecular characterization of a novel metabotropic glutamate receptor mGluR5 coupled to inositol phosphate/Ca2+ signal transduction. J. Biol. Chem. 267, 13361-13368. doi: 10.1016/S0021-9258(18)42219-3

Agulhon, C., Fiacco, T. A., and Mccarthy, K. D. (2010). Hippocampal short- and long-term plasticity are not modulated by astrocyte Ca2+ signaling. Science 327, 1250-1254. doi: 10.1126/science.1184821

Alba, A., Kano, M., Chong, C., Stanton, M. E., Fox, G. D., Herrup, K., et al. (1994). Deficient cerebellar long-term depression and impaired motor learning in mGluR1 mutant mice. Cell 79:377. doi: 10.1016/0092-8674(94)90205-4

Allen, N. J., Bennett, M. L., Foo, L. C., Wang, G. X., Chakraborty, C., Smith, S. J., et al. (2012). Astrocyte glypicans 4 and 6 promote formation of excitatory synapses via GluA1 AMPA receptors. Nature 486, 410-414. doi: 10.1038/nature11059

Amir, R. E., Van, D., Wan, M., Tran, C. Q., Francke, U., and Zoghbi, H. Y. (1999). Rett syndrome is caused by mutations in X-linked MECP2, encoding methyl-CpG-binding protein 2. Nat. Genet. 23, 185-188. doi: 10.1038/13810

Araque, A., Carmignoto, G., Haydon, P. G., Oliet, S. H., Robitaille, R., and Volterra, A. (2014). Gliotransmitters travel in time and space. Neuron 81, 728-739. doi: 10.1016/j.neuron.2014.02.007

Araque, A., Martín, E. D., Perea, G., Arellano, J. I., and Buño, W. (2002). Synaptically released acetylcholine evokes $\mathrm{Ca} 2+$ elevations in astrocytes in hippocampal slices. J. Neurosci. 22, 2443-2450. doi: 10.1523/JNEUROSCI.22-07-02443. 2002

Araque, A., Parpura, V., Sanzgiri, R. P., and Haydon, P. G. (1999). Tripartite synapses: glia, the unacknowledged partner. Trends Neurosci. 22, 208-215. doi: 10.1016/S0166-2236(98)01349-6

Azkue, J. J., Murga, M., Fernández-Capetillo, O., Mateos, J. M., and Grandes, P. (2015). Immunoreactivity for the group III metabotropic glutamate receptor subtype mGluR4a in the superficial laminae of the rat spinal dorsal horn. J. Compar. Neurol. 430, 448-457. 10.1002/10969861(20010219)430:4<448::AID-CNE1042>3.0.CO;2-O

Bae, J. J., Xiang, Y. Y., Martinez-Canabal, A., Frankland, P. W., Yang, B. B., and Lu, W. Y. (2011). Increased transforming growth factor-betal modulates not only enhance our understanding of healthy synaptic development, but also guide the search for new treatments against neurodegenerative diseases.

\section{AUTHOR CONTRIBUTIONS}

$\mathrm{XL}$ and $\mathrm{FH}$ contributed in study design, literature search, and manuscript preparation. JY and XW helped conceptualize, write the original draft, and review and edit the manuscript. QZ, TZ, SY, WY, DY, and YF helped review and edit the manuscript. This manuscript was handled by XL. All authors contributed to the article and approved the submitted version.

\section{FUNDING}

This work was supported by grants from the National Natural Science Foundation of China (81760261 and 82060219); Provincal Science foudation of Jiangxi (20192BCB23024 and 20202BABL206016); Youth Team Project of the Second Affiliated Hospital of Nanchang University (2019YNTD12003). glutamate receptor expression in the hippocampus. Int. J. Physiol. Pathophysiol. Pharmacol. 3, 9-20.

Baldwin, K. T., and Eroglu, C. (2017). Molecular mechanisms of astrocyte-induced synaptogenesis. Curr. Opin. Neurobiol. 45, 113-120. doi: 10.1016/j.conb.2017.05.006

Bandtlow, C. E., and Zimmermann, D. R. (2000). Proteoglycans in the developing brain: new conceptual insights for old proteins. Physiol. Rev. 80, 1267-1290. doi: 10.1152/physrev.2000.80.4.1267

Baudouin, S., and Scheiffele, P. (2010). SnapShot: neuroligin-neurexin complexes. Cell 141:908.e1. doi: 10.1016/j.cell.2010.05.024

Bazenet, C. E., and Rubin, M. (1998). The small GTP-binding protein Cdc42 is required for nerve growth factor withdrawal-induced neuronal death. Proc. Natl. Acad. Sci. U. S. A. 95, 3984-3989. doi: 10.1073/pnas.95.7.3984

Bear, M. F., Huber, K. M., and Warren, S. T. (2004). The mGluR theory of fragile X mental retardation. Trends Neurosci. 27, 370-377. doi: 10.1016/j.tins.2004.04.009

Beenhakker, M. P., and Huguenard, J. R. (2010). Astrocytes as gatekeepers of GABAB receptor function. J. Neurosci. 30:15262. doi: 10.1523/JNEUROSCI.3243-10.2010

Benavides-Piccione, R., Ballesteros-Yáñez, I., de Lagrán, M. M., Elston, G., Estivill, X., Fillat, C., et al. (2004). On dendrites in down syndrome and DS murine models: a spiny way to learn. Prog Neurobiol. 74, 111-126. doi: 10.1016/j.pneurobio.2004.08.001

Benneyworth, M. A., Li, Y., Basu, A. C., Bolshakov, V. Y., and Coyle, J. T. (2012). Cell selective conditional null mutations of serine racemase demonstrate a predominate localization in cortical glutamatergic neurons. Cell. Mol. Neurobiol. 32, 613-624. doi: 10.1007/s10571-012-9808-4

Benquet, P., Gee, C. E., and Gerber, U. (2002). Two distinct signaling pathways upregulate NMDA receptor responses via two distinct metabotropic glutamate receptor subtypes. J. Neurosci. 22, 9679-9686. doi: 10.1523/JNEUROSCI.22-22-09679.2002

Berretta, N., Irving, A. J., Seal, A. J., Henley, J. M., Jane, D. E., Watkins, J. C., et al. (1993). Induction of LTP in the hippocampus needs synaptic activation of glutamate metabotropic receptors. Nature 363, 347-350. doi: 10.1038/363347a0

Bialas, A. R., and Stevens, B. (2013). TGF-beta signaling regulates neuronal C1q expression and developmental synaptic refinement. Nat. Neurosci. 16, 1773-1782. doi: 10.1038/nn.3560 
Bianchi, R., Chuang, S. C., Zhao, W., Young, S. R., and Wong, R. (2009). Cellular plasticity for Group I mGluR-mediated epileptogenesis. J. Neurosci. 29:3497. doi: 10.1523/JNEUROSCI.5447-08.2009

Biber, K., Laurie, D. J., Berthele, A., Sommer, B., Tölle, T., Gebicke-Härter, P., et al. (2010). Expression and signaling of group I metabotropic glutamate receptors in astrocytes and microglia. J. Neurochem. 72, 1671-1680. doi: 10.1046/j.1471-4159.1999.721671.x

Billard, J. M. (2012). d-Amino acids in brain neurotransmission and synaptic plasticity. Amino Acids. 43, 1851-1860. doi: 10.1007/s00726-012-1346-3

Birnbaumer, L. (2009). The TRPC class of ion channels: a critical review of their roles in slow, sustained increases in intracellular $\mathrm{Ca}(2+)$ concentrations. Ann. Rev. Pharmacol. Toxicol. 49:395. doi: 10.1146/annurev.pharmtox.48.113006.094928

Brill, J., and Huguenard, J. R. (2008). Sequential changes in AMPA receptor targeting in the developing neocortical excitatory circuit. J. Neurosci. 28, 13918-13928. doi: 10.1523/JNEUROSCI.3229-08.2008

Burnstock, G., and Kennedy, C. (2011). P2X receptors in health and disease. Adv. Pharmacol. 61:333. doi: 10.1016/B978-0-12-385526-8.00011-4

Caraci, F., Gulisano, W., Guida, C. A., Impellizzeri, A. A. R., Drago, F., Puzzo, D., et al. (2015). A key role for TGF- $\beta 1$ in hippocampal synaptic plasticity and memory. Sci. Rep. 5:11252. doi: 10.1038/srep11252

Caraci, F., Spampinato, S. F., Morgese, M. G., Tascedda, F., Salluzzo, M. G., Giambirtone, M. C., et al. (2018). Neurobiological links between depression and $\mathrm{AD}$ : the role of TGF- $\beta 1$ signaling as a new pharmacological target. Pharmacol. Res. 130, 374-384. doi: 10.1016/j.phrs.2018.02.007

Caselli, R. J., Reiman, E. M., Osborne, D., Hentz, J. G., Baxter, L. C., Hernandez, J. L., et al. (2004). Longitudinal changes in cognition and behavior in asymptomatic carriers of the APOE e4 allele. Neurology 62:1990. doi: 10.1212/01.WNL.0000129533.26544.BF

Castillo, P. E., Malenka, R. C., and Nicoll, R. A. (1997). Kainate receptors mediate a slow postsynaptic current in hippocampal CA3 neurons. Nature 388, 182-186. doi: $10.1038 / 40645$

Castro, M., Chuquet, J., Liaudet, N., Bhaukaurally, K., Santello, M., Bouvier, D., et al. (2011). Local Ca2+ detection and modulation of synaptic release by astrocytes. Nat. Neurosci. 14:1276. doi: 10.1038/nn.2929

Chang, Q., Khare, G., Dani, V., Nelson, S., and Jaenisch, R. (2006). The disease progression of Mecp2 mutant mice is affected by the level of BDNF expression. Neuron 49, 341-348. doi: 10.1016/j.neuron.2005.12.027

Chao, M. V., and Bothwell, M. (2002). Neurotrophins: to cleave or not to cleave. Neuron 33, 9-12. doi: 10.1016/S0896-6273(01)00573-6

Chen, J., Tan, Z., Zeng, L., Zhang, X., He, Y., Gao, W., et al. (2013). Heterosynaptic long-term depression mediated by ATP released from astrocytes. Glia 61, 178-191. doi: 10.1002/glia.22425

Cho, K., Kemp, N., Noel, J., Aggleton, J. P., and Bashir, Z. I. (2000). A new form of long-term depression in the perirhinal cortex. Nat. Neurosci 3:150. doi: $10.1038 / 72093$

Choquet, D. (2018). Linking nanoscale dynamics of AMPA receptor organization to plasticity of excitatory synapses and learning. J. Neurosci. 38, 9318-9329. doi: 10.1523/JNEUROSCI.2119-18.2018

Christopher, R. W., Namsoo, K., Sehwon, K., Ji-Eun, C., Petar, M., Spence, E. F., et al. (2018). Thrombospondin receptor $\alpha 2 \delta-1$ promotes synaptogenesis and spinogenesis via postsynaptic Rac1. J. Cell Biol. 217:jcb.201802057. doi: $10.1083 /$ jcb.201802057

Christopherson, K. S., Ullian, E. M., Stokes, C. C., Mullowney, C. E., Hell, J. W., Agah, A., et al. (2005). Thrombospondins are astrocyte-secreted proteins that promote CNS synaptogenesis. Cell 120, 421-433. doi: 10.1016/j.cell.2004.12.020

Chuang, S.-C. (2005). Prolonged epileptiform discharges induced by altered group I metabotropic glutamate receptor-mediated synaptic responses in hippocampal slices of a fragile X mouse model. J. Neurosci. 25, 8048-8055. doi: 10.1523/JNEUROSCI.1777-05.2005

Chung, W. S., Allen, N. J., and Eroglu, C. (2015). Astrocytes control synapse formation, function, and elimination. Cold Spring Harb. Perspect. Biol. 7:a020370. doi: 10.1101/cshperspect.a020370

Chung, W. S., Clarke, L. E., Wang, G. X., Stafford, B. K., Sher, A., Chakraborty, C., et al. (2013). Astrocytes mediate synapse elimination through MEGF10 and MERTK pathways. Nature 504, 394-400. doi: 10.1038/nature12776

Clarke, L. E., and Barres, B. A. (2013). Glia keep synapse distribution under wraps. Cell. 154, 267-268. doi: 10.1016/j.cell.2013.06.045
Comery, T. A., Harris, J. B., Willems, P. J., Oostra, B. A., Irwin, S. A., Weiler, I. J., et al. (1997). Abnormal dendritic spines in fragile X knockout mice: maturation and pruning deficits. Proc. Natl. Acad. Sci. U. S. A. 94, 5401-5404. doi: $10.1073 /$ pnas. 94.10 .5401

Contractor, A., Klyachko, V., and Portera-Cailliau, C. (2015). Altered neuronal and circuit excitability in fragile X syndrome. Neuron 87, 699-715. doi: 10.1016/j.neuron.2015.06.017

Cornell-Bell, A., Finkbeiner, S., Cooper, M., and Smith, S. (1990). Glutamate induces calcium waves in cultured astrocytes: long-range glial signaling. Science. 247, 470-473. doi: 10.1126/science.1967852

Corti, C., Restituito, S., Rimland, J. M., Brabet, I., Corsi, M., Pin, J. P., et al. (1998). Cloning and characterization of alternative mRNA forms for the rat metabotropic glutamate receptors mGluR7 and mGluR8. Eur. J. Neurosc. 10, 3629-3641. doi: 10.1046/j.1460-9568.1998.00371.x

Crosby, K. M., Murphy-Royal, C., Wilson, S. A., Gordon, G. R., Bains, J. S., and Pittman, Q. J. (2018). Cholecystokinin switches the plasticity of GABA synapses in the dorsomedial hypothalamus via astrocytic ATP release. J. Neurosci. 38, 8515-8525. doi: 10.1523/JNEUROSCI.0569-18.2018

Dailey, M. E., and Smith, S. J. (1996). The dynamics of dendritic structure in developing hippocampal slices. J. Neurosci. 16, 2983-2994. doi: 10.1523/JNEUROSCI.16-09-02983.1996

Daniel, H., Ferraguti, F., Bordi, F., Franz-Bacon, K., Reggiani, A., Matarese, V., et al. (1994). Motor deficit and impairment of synaptic plasticity in mice lacking mGluR1. Nature 372, 237-243. doi: 10.1038/372237a0

Defreitas, M. F., Mcquillen, P. S., and Shatz, C. J. (2001). A novel p75NTR signaling pathway promotes survival, not death, of immunopurified neocortical subplate neurons. J. Neurosci. 21, 5121-5129. doi: 10.1523/JNEUROSCI.21-14-05121.2001

Diniz, L. P., Tortelli, V., Garcia, M. N., Araújo, A. P. B., Melo, H. M., Seixas d, S., et al. (2015). Astrocyte transforming growth factor beta 1 promotes inhibitory synapse formation via CaM kinase II signaling. Glia 62, 1917-1931. doi: $10.1002 /$ glia.22713

Duan, G. F., Ye, Y., Xu, S., Tao, W., Zhao, S., Jin, T., et al. (2018). Signal peptide represses GluK1 surface and synaptic trafficking through binding to aminoterminal domain. Nat. Commun. 9, 1-11. doi: 10.1038/s41467-018-07403-7

Dubois, C. J., Lachamp, P. M., Sun, L., Mishina, M., and Liu, S. J. (2016). Presynaptic GluN2D receptors detect glutamate spillover and regulate cerebellar GABA release. J. Neurophysiol. 115, 271-285. doi: $10.1152 /$ jn. 00687.2015

Duvoisin, R. M., Zhang, C., and Ramonell, K. (1995). A novel metabotropic glutamate receptor expressed in the retina and olfactory bulb. J. Neurosci. 15:3075. doi: 10.1523/JNEUROSCI.15-04-03075.1995

Dzyubenko, E., Gottschling, C., and Faissner, A. (2016). Neuron-glia interactions in neural plasticity: contributions of neural extracellular matrix and perineuronal nets. Neural Plast. 2016:5214961. doi: 10.1155/2016/5214961

Eroglu, C., Allen, N. J., Susman, M. W., O’Rourke, N. A., and Park, C. Y., Özkan, E., et al. (2009). Gabapentin receptor alpha2delta-1 is a neuronal thrombospondin receptor responsible for excitatory CNS synaptogenesis. Cell 139, 380-392. doi: 10.1016/j.cell.2009.09.025

Faludi, G., and Mirnics, K. (2011). Synaptic changes in the brain of subjects with schizophrenia. Int. J. Dev. Neurosci. 29, 305-309. doi: 10.1016/j.ijdevneu.2011.02.013

Farhy-Tselnicker, I., van Casteren, A. C., Lee, A., Chang, V. T., Aricescu, A. R., and Allen, N. J. (2017). Astrocyte-secreted glypican 4 regulates release of neuronal pentraxin 1 from axons to induce functional synapse formation. Neuron 96, 428-445. doi: 10.1016/j.neuron.2017.09.053

Fattorini, G., Ripoli, C., Cocco, S., Spinelli, M., Mattera, A., Grassi, C., et al. (2019). Glutamate/GABA co-release selectively influences postsynaptic glutamate receptors in mouse cortical neurons. Neuropharmacology 161:107737. doi: 10.1016/j.neuropharm.2019.107737

Feng, P., Aldridge, G. M., Greenough, W. T., and Gan, W. B. (2010). Dendritic spine instability and insensitivity to modulation by sensory experience in a mouse model of fragile X syndrome. Proc. Natl. Acad. Sci. U. S. A. 107, 17768-17773. doi: 10.1073/pnas.1012496107

Fitzjohna, S. M., Irving, A. J., Palmer, M. J., Harvey, J., Lodge, D., and Collingridge, G. L. (1996). Activation of group I mGluRs potentiates NMDA responses in rat hippocampal slices. Neurosci. Lett. 203, 211-213. doi: 10.1016/0304-3940(96)12301-6 
Fotuhi, M., Standaert, D. G., Testa, C. M., Penney John, and B., Young, A. B. (1994). Differential expression of metabotropic glutamate receptors in the hippocampus and entorhinal cortex of the rat. Brain Res. Mol. Brain Res. 21, 283-292. doi: 10.1016/0169-328X(94)90259-3

Frerking, M., Malenka, R. C., and Nicoll, R. A. (1998). Synaptic activation of kainate receptors on hippocampal interneurons. Nat. Neurosci. 1, 479-486. doi: $10.1038 / 2194$

Garrett, A. M., and Weiner, J. A. (2009). Control of CNS synapse development by $\{$ gamma\}-protocadherin-mediated astrocyte-neuron contact. J. Neurosci. 29:11723. doi: 10.1523/JNEUROSCI.2818-09.2009

Gerber, U., Gee, C. E., and Benquet, P. (2007). Metabotropic glutamate receptors: intracellular signaling pathways. Curr. Opin. Pharmacol. 7, 56-61. doi: 10.1016/j.coph.2006.08.008

Grishin, A. A. (2004). Differential calcium-dependent modulation of NMDA currents in CA1 and CA3 hippocampal pyramidal cells. J. Neurosci. 24, 350-355. doi: 10.1523/JNEUROSCI.4933-03.2004

Grosche, J., Matyash, V., Möller, T., Verkhratsky, A., Reichenbach, A., and Kettenmann, H. (1999). Microdomains for neuron-glia interaction: parallel fiber signaling to Bergmann glial cells. Nat. Neurosci. 2, 139-143. doi: $10.1038 / 5692$

Grunseich, C., Mandel, G., Ballas, N., and Lioy, D. T. (2009). Non-cell autonomous influence of MeCP2-deficient glia on neuronal dendritic morphology. Nat. Neurosci. 12:311. doi: 10.1038/nn.2275

Grunwald, I. C., Korte, M., Adelmann, G., Plueck, A., Kullander, K., Adams, R. H., et al. (2004). Hippocampal plasticity requires postsynaptic ephrinBs. Nat. Neurosci. 7, 33-40. doi: 10.1038/nn1164

Hagit, S., Marie, B. J., Paul, S. G., Hazem, S., Samah, N., Eitan, K., et al. (2016). Asc1 transporter regulation of synaptic activity via the tonic release of $\mathrm{d}$-serine in the forebrain. Cerebral Cortex. 27:1573. doi: 10.1093/cercor/bhv350

Halassa, M. M., and Haydon, P. G. (2010). Integrated brain circuits: astrocytic networks modulate neuronal activity and behavior. Annu. Rev. Physiol. 72, 335-355. doi: 10.1146/annurev-physiol-021909-135843

Hamada, K., and Mikoshiba, K. (2020). IP3 receptor plasticity underlying diverse functions. Annu. Rev. Physiol. 82, 151-176. doi: 10.1146/annurev-physiol-021119-034433

Hanisch, U. K., and Kettenmann, H. (2007). Microglia: active sensor and versatile effector cells in the normal and pathologic brain. Nat. Neurosci. 10, 1387-1394. doi: $10.1038 / \mathrm{nn} 1997$

Henkemeyer, M., Itkis, O. S., Ngo, M., Hickmott, P. W., and Ethell, I. M. (2003). Multiple EphB receptor tyrosine kinases shape dendritic spines in the hippocampus. J. Cell Biol. 163, 1313-1326. doi: 10.1083/jcb.200306033

Henley, J. M., and Wilkinson, K. A. (2016). Synaptic AMPA receptor composition in development, plasticity and disease. Nat. Rev. Neurosci. 17, 337-350. doi: 10.1038/nrn.2016.37

Henneberger, C., Papouin, T., Oliet, S. H., and Rusakov, D. A. (2010). Longterm potentiation depends on release of D-serine from astrocytes. Nature 463, 232-236. doi: 10.1038/nature08673

Higashimori, H., Schin, C. S., Chiang, M., Morel, L., Shoneye, T. A., Nelson, D. L., et al. (2016). Selective deletion of astroglial FMRP dysregulates glutamate transporter GLT1 and contributes to fragile X syndrome phenotypes in vivo. J. Neurosci. 36, 7079-7094. doi: 10.1523/JNEUROSCI.1069-16.2016

Hodges, J. L., Yu, X., Gilmore, A., Bennett, H., Tjia, M., Perna, J. F., et al. (2016). Astrocytic contributions to synaptic and learning abnormalities in a mouse model of fragile X syndrome. Biol. Psychiatry. 82:139. doi: 10.1016/j.biopsych.2016.08.036

Holt, L. M., Hernandez, R. D., Pacheco, N. L., Ceja, B. T., and Olsen, M. L. (2019). Astrocyte morphogenesis is dependent on BDNF signaling via astrocytic TrkB.T1. eLife Sci. 8: e44667. doi: 10.7554/eLife.44667

Huber, K. M., Gallagher, S. M., and Warren, S. T. (2002). Altered synaptic plasticity in a mouse model of fragile X mental retardation. Proc. Natl. Acad. Sci. U. S. A. 99, 7746-7750. doi: 10.1073/pnas.122205699

Iossifov, I., O’Roak, B. J., Sanders, S. J., Ronemus, M., and Wigler, M. (2014). The contribution of de novo coding mutations to autism spectrum disorder. Nature 515, 216-221. doi: 10.1038/nature13908

Irwin, S. A., Patel, B., Idupulapati, M., Harris, J. B., Crisostomo, R. A., Larsen, B. P., et al. (2001). Abnormal dendritic spine characteristics in the temporal and visual cortices of patients with fragile-X syndrome: a quantitative examination. Am. J. Med. Genet. 98, 161-167. doi: 10.1002/1096-8628(20010115)98:2<161::AID-AJMG1025>3.0.CO;2-B

Jo, S., Yarishkin, O., Hwang, Y. J., Chun, Y. E., Park, M., Woo, D. H., et al. (2014). GABA from reactive astrocytes impairs memory in mouse models of Alzheimer's disease. Nat. Med. 20, 886-896. doi: 10.1038/nm.3639

John, N., Krügel, H., Frischknecht, R., Smalla, K. H., Schultz, C., Kreutz, M. R., et al. (2006). Brevican-containing perineuronal nets of extracellular matrix in dissociated hippocampal primary cultures. Mol. Cell. Neurosci. 31, 774-784. doi: 10.1016/j.mcn.2006.01.011

Jon, P. F., Isabel, A. M., and Juan, L. (2016). Synaptic targeting of kainate receptors. Cerebral Cortex. 26:1464. doi: 10.1093/cercor/bhu244

Jourdain, P., Bergersen, L. H., Bhaukaurally, K., Bezzi, P., Santello, M., Domercq, M., et al. (2007). Glutamate exocytosis from astrocytes controls synaptic strength. Nat. Neurosci. 10, 331-339. doi: 10.1038/nn1849

Kaczor, P. T., and Mozrzymas, J. W. (2017). Key metabolic enzymes underlying astrocytic upregulation of GABAergic plasticity. Front. Cell. Neurosci. 11:144. doi: 10.3389/fncel.2017.00144

Kanemaru, K., Sekiya, H., Xu, M., Satoh, K., Kitajima, N., Yoshida, K., et al. (2014). In vivo visualization of subtle, transient, and local activity of astrocytes using an ultrasensitive Ca2+ indicator. Cell Rep. 8, 311-318. doi: 10.1016/j.celrep.2014.05.056

Kang, J., Jiang, L., Goldman, S. A., and Nedergaard, M. (1998). Astrocyte-mediated potentiation of inhibitory synaptic transmission. Nat. Neurosci. 1, 683-692. doi: $10.1038 / 3684$

Kierdorf, K., and Prinz, M. (2013). Factors regulating microglia activation. Front. Cell. Neurosci. 7:44. doi: 10.3389/fncel.2013.00044

Kinoshita, A., Ohishi, H., Nomura, S., Shigemoto, R., Nakanishi, S., Mizuno, N., et al. (1996). Presynaptic localization of a metabotropic glutamate receptor, mGluR4a, in the cerebellar cortex: a light and electron microscope study in the rat. Neurosci. Lett. 207, 199-202. doi: 10.1016/0304-3940(96)12519-2

Kinoshita, A., Shigemoto, R., Ohishi, H., Putten, H., and Mizuno, N. (2015). Immunohistochemical localization of metabotropic glutamate receptors, mGluR7a and mGluR7b, in the central nervous system of the adult rat and mouse: a light and electron microscopic study. J. Compar. Neurol. 393, 332-352. doi: 10.1002/(SICI)1096-9861(19980413)393:3 <332::AID-CNE6>3.0.CO;2-2

Kniazeff, J., Prézeau, L., Rondard, P., Pin, J. P., and Goudet, C. (2011). Dimers and beyond: the functional puzzles of class C GPCRs. Pharmacol. Ther. 130, 9-25. doi: 10.1016/j.pharmthera.2011.01.006

Koekkoek, S. K., Yamaguchi, K., Milojkovic, B. A., Dortland, B. R., and De Zeeuw, C. I. (2005). Deletion of FMR1 in Purkinje cells enhances parallel fiber LTD, enlarges spines, and attenuates cerebellar eyelid conditioning in Fragile X syndrome. Neuron 47, 339-352. doi: 10.1016/j.neuron.2005. 07.005

Koeppen, J., Nguyen, A. Q., Nikolakopoulou, A. M., Garcia, M., Hanna, S., Woodruff, S., et al. (2018). Functional consequences of synapse remodeling following astrocyte-specific regulation of Ephrin-B1 in the adult hippocampus. J. Neurosci. 38, 5710-5726. doi: 10.1523/JNEUROSCI.3618-17.2018

Kucukdereli, H., Allen, N. J., Lee, A. T., Feng, A., Ozlu, M. I., Conatser, L. M., et al. (2011). Control of excitatory CNS synaptogenesis by astrocyte-secreted proteins Hevin and SPARC. Proc. Natl. Acad. Sci. U. S. A. 108, E440-E449. doi: 10.1073/pnas.1104977108

Kwon, H. B., and Sabatini, B. L. (2011). Glutamate induces de novo growth of functional spines in developing cortex. Nature 474:100. doi: $10.1038 /$ nature 09986

Lai, M. C., Lombardo, M. V., and Baron-Cohen, S. (2014). Autism. Lancet 383, 896-910. doi: 10.1016/S0140-6736(13)61539-1

Lalo, U., Bogdanov, A., and Pankratov, Y. (2019). Age- and experience-related plasticity of ATP-mediated signaling in the neocortex. Front. Cell. Neurosci. 13:242. doi: 10.3389/fncel.2019.00242

Lan, J. Y., Skeberdis, V. A., Jover, T., Zheng, X., Bennett, M., and Zukin, R. S. (2001). Activation of metabotropic glutamate receptor 1 accelerates NMDA receptor trafficking. J. Neurosci. 21, 6058-6068. doi: 10.1523/JNEUROSCI.21-16-06058.2001

Lau, L. W., Cua, R., Keough, M. B., Haylock-Jacobs, S., and Yong, V. W. (2013). Pathophysiology of the brain extracellular matrix: a new target for remyelination. Nat. Rev. Neurosci. 14, 722-729. doi: 10.1038/ nrn3550 
Lee, S., Yoon, B. E., Berglund, K., Oh, S. J., Park, H., Shin, H. S., et al. (2010). Channel-mediated tonic GABA release from glia. Science 330, 790-796. doi: $10.1126 /$ science. 1184334

Lerma, J. (2006). Kainate receptor physiology. Curr. Opin. Pharmacol. 6, 89-97. doi: 10.1016/j.coph.2005.08.004

Lerma, J. (2011). Net(o) excitement for kainate receptors. Nat. Neurosci. 14, 808-810. doi: 10.1038/nn.2864

Lerma, J., and Marques, J. M. (2013). Kainate receptors in health and disease. Neuron 80, 292-311. doi: 10.1016/j.neuron.2013.09.045

Lerma, J., Paternain, A. V., Rodríguez-Moreno, A., and López-García, J. C. (2001). Molecular physiology of kainate receptors. Physiol. Rev. 81:971. doi: 10.1152/physrev.2001.81.3.971

Lewis, P., Abbeduto, L., Murphy, M., Richmond, E., and Schroeder, S. (2010). Cognitive, language and social-cognitive skills of individuals with fragile $\mathrm{X}$ syndrome with and without autism. J. Intellect. Disabil. Res. 50, 532-545. doi: 10.1111/j.1365-2788.2006.00803.x

Li, X., Zhang, C., Bian, Q., Gao, N., Zhang, X., Meng, Q., et al. (2016). Integrative functional transcriptomic analyses implicate specific molecular pathways in pulmonary toxicity from exposure to aluminum oxide nanoparticles. Nanotoxicology 10, 957-969. doi: 10.3109/17435390.2016.1149632

Linggi, M. S., Burke, T. L., Williams, B. B., Harrington, A., Kraemer, R., Hempstead, B. L., et al. (2005). Neurotrophin receptor interacting factor. (NRIF) is an essential mediator of apoptotic signaling by the p75 neurotrophin receptor. J. Biol. Chem. 280, 13801-13808. doi: 10.1074/jbc.M410435200

Lioy, D. T., Garg, S. K., Monaghan, C. E., Raber, J., Foust, K. D., Kaspar, B. K., et al. (2011). A role for glia in the progression of Rett's syndrome. Nature 475, 497-500. doi: 10.1038/nature 10214

Lu, B. (2003). BDNF and activity-dependent synaptic modulation. Learn. Mem. 10, 86-98. doi: $10.1101 / \mathrm{lm} .54603$

Lu, Y. M., Jia, Z., Janus, C., Henderson, J. T., Gerlai, R., Wojtowicz, J. M., et al. (1997). Mice lacking metabotropic glutamate receptor 5 show impaired learning and reduced CA1 long-term potentiation. (LTP) but normal CA3 LTP. J. Neurosci. 17, 5196-5205. doi: 10.1523/JNEUROSCI.17-13-05196.1997

Maezawa, I., Swanberg, S., Harvey, D., Lasalle, J. M., and Jin, L. W. (2009). Rett syndrome astrocytes are abnormal and spread MeCP2 deficiency through gap junctions. J. Neurosci. 29, 5051-5061. doi: 10.1523/JNEUROSCI.0324-09.2009

Makino, H., and Malinow, R. (2009). AMPA receptor incorporation into synapses during LTP: the role of lateral movement and exocytosis. Neuron 64, 381-390. doi: $10.1016 /$ j.neuron.2009.08.035

Makoff, A., Volpe, F., Lelchuk, R., Harrington, K., and Emson, P. (1996). Molecular characterization and localization of human metabotropic glutamate receptor type 3. Mol. Brain Res. 40, 0-63. doi: 10.1016/0169-328X(96)00037-X

Marin-Padilla, M. (1972). Structural abnormalities of the cerebral cortex in human chromosomal aberrations: a Golgi study. Brain Res. 66, 375-391. doi: 10.1016/0006-8993(74)90054-7

Mariotti, L., Losi, G., Sessolo, M., Marcon, I., and Carmignoto, G. (2015). The inhibitory neurotransmitter GABA evokes long-lasting Ca2+oscillations in cortical astrocytes. Glia 64, 363-373. doi: 10.1002/glia. 22933

Masuoka, T., Ikeda, R., and Konishi, S. (2019). Persistent activation of histamine $\mathrm{H} 1$ receptors in the hippocampal CA1 region enhances NMDA receptor-mediated synaptic excitation and long-term potentiation in astrocyte- and D-serine-dependent manner. Neuropharmacology 151, 64-73. doi: 10.1016/j.neuropharm.2019.03.036

Mckinney, B. C., Grossman, A. W., Elisseou, N. M., and Greenough, W. T. (2010). Dendritic spine abnormalities in the occipital cortex of C57BL/6 Fmr1 knockout mice. Am. J. Med. Genet. Part B Neuropsychiatr. Genet. 136B, 98-102. doi: 10.1002/ajmg.b.30183

Middleton, G. (2000). Cytokine-induced nuclear factor kappa B activation promotes the survival of developing neurons. J. Cell Biol. 148, 325-332. doi: $10.1083 /$ jcb.148.2.325

Miller, S., Romano, C., and Cotman, C. W. (1995). Growth factor upregulation of a phosphoinositide-coupled metabotropic glutamate receptor in cortical astrocytes. J. Neurosci. 15:6103. doi: 10.1523/JNEUROSCI.15-09-06103.1995

Mohapatra, J., Sharma, M., Acharya, A., Pandya, G., and Jain, M. R. (2011). Guide to receptors and channels. (GRAC), 3rd edition. (2008 revision). Br. J. Pharmacol. 164, S1-324. doi: 10.1111/j.1476-5381.2011.01649_1.x
Morgan, J. T., Chana, G., Pardo, C. A., Achim, C., Semendeferi, K., Buckwalter, J., et al. (2010). Microglial activation and increased microglial density observed in the dorsolateral prefrontal cortex in autism. Biol. Psychiatry. 68, 368-376. doi: 10.1016/j.biopsych.2010.05.024

Moult, P. R. (2006). Tyrosine phosphatases regulate AMPA receptor trafficking during metabotropic glutamate receptor-mediated long-term depression. J. Neurosci. 26:2544. doi: 10.1523/JNEUROSCI.4322-05.2006

Muller, M. S., and Taylor, C. W. (2017). ATP evokes $\mathrm{Ca}(2+)$ signals in cultured foetal human cortical astrocytes entirely through G protein-coupled P2Y receptors. J. Neurochem. 142, 876-885. doi: 10.1111/jnc.14119

Muthukumar, A. K., Stork, T., and Freeman, M. R. (2014). Activity-dependent regulation of astrocyte GAT levels during synaptogenesis. Nat. Neurosci. 17, 1340-1350. doi: 10.1038/nn.3791

Nakajima, Y., Iwakabe, H., Akazawa, C., Nawa, H., and Nakanishi, S. (1993). Molecular characterization of a novel retinal metabotropic glutamate receptor mglurg with a high agonist selectivity for L-2-amino-4-phosphonobutyrate. J. Biol. Chem. 268, 11868-11873. doi: 10.1016/S0021-9258(19)50280-0

Nakamoto, M., Nalavadi, V., Epstein, M. P., Narayanan, U., Bassell, G. J., and Warren, S. T. (2007). Fragile X mental retardation protein deficiency leads to excessive mGluR5-dependent internalization of AMPA receptors. Proc. Nat. Acad. Sci. 104, 15537-15542. doi: 10.1073/pnas.0707484104

Nett, W. J., Oloff, S. H., and Mccarthy, K. D. (2002). Hippocampal astrocytes in situ exhibit calcium oscillations that occur independent of neuronal activity. J. Neurophysiol. 87, 528-537. doi: 10.1152/jn.00268.2001

Newton, R. A., Bingham, S., Case, P. C., Sanger, G. J., and Lawson, S. N. (2001). Dorsal root ganglion neurons show increased expression of the calcium channel alpha2delta-1 subunit following partial sciatic nerve injury. Mol. Brain Res. 95, 1-8. doi: 10.1016/S0169-328X(01)00188-7

Nguyen, P. T., Dorman, L. C., Pan, S., Vainchtein, I. D., Han, R. T., Nakao-Inoue, H., et al. (2020). Microglial remodeling of the extracellular matrix promotes synapse plasticity. Cell 182, 388-403.e15. doi: 10.1016/j.cell.2020.05.050

Nimmerjahn, A., Mukamel, E. A., and Schnitzer, M. J. (2009). Motor behavior activates Bergmann glial networks. Neuron 62, 400-412. doi: 10.1016/j.neuron.2009.03.019

Norio, T., Tsuneko, M., Chihiro, H., Terumi, N., Etsuko, E., Katsuhiko, M., et al. (2011). Astrocyte calcium signaling transforms cholinergic modulation to cortical plasticity in vivo. Neurosci. Res. 31, 18155-18165. doi: 10.1523/JNEUROSCI.5289-11.2011

Octavio, G., Maria, T., Pablo, H., Pinar, C., Jorge, B., and Fe Any, M. B. (2010). A role for thrombospondin-1 deficits in astrocyte-mediated spine and synaptic pathology in down's syndrome. PLoS ONE. 5:e14200. doi: 10.1371/journal.pone.0014200

Ohishi, H., Ogawa-Meguro, R., Shigemoto, R., Kaneko, T., and Mizuno, N. (1994). Immunohistochemical localization of metabotropic glutamate receptors, mGluR2 and mGluR3, in rat cerebellar cortex. Neuron 13, 55-66. doi: 10.1016/0896-6273(94)90459-6

Ohishi, H., Shigemoto, R., Nakanishi, S., and Mizuno, N. (1993). Distribution of the messenger RNA for a metabotropic glutamate receptor, mGluR2, in the central nervous system of the rat. Neuroscience 53, 1009-1018. doi: 10.1016/0306-4522(93)9 0485-X

Ohishi, H., Shigemoto, R., Nakanishi, S., and Mizuno, N. (2010). Distribution of the mRNA for a metabotropic glutamate receptor. (mGluR3) in the rat brain: an in situ hybridization study. J. Compar. Neurol. 335, 252-266. doi: 10.1002/cne.903350209

Opazo, P., and Choquet, D. (2011). A three-step model for the synaptic recruitment of AMPA receptors. Mol. Cell. Neurosci. 46, 1-8. doi: 10.1016/j.men.2010.08.014

Padmashri, R., Reiner, B. C., Suresh, A., Spartz, E., and Dunaevsky, A. (2011). Altered structural and functional synaptic plasticity with motor skill learning in a mouse model of fragile X syndrome. J. Neurosci. 33:19715. doi: 10.1523/JNEUROSCI.2514-13.2013

Panatier, A., Vallee, J., Haber, M., Murai, K. K., Lacaille, J. C., and Robitaille, R. (2011). Astrocytes are endogenous regulators of basal transmission at central synapses. Cell 146, 785-798. doi: 10.1016/j.cell.2011.07.022

Panja, D., Kenney, J. W., D’Andrea, L., Zalfa, F., Vedeler, A., Wibrand, K., et al. (2014). Two-stage translational control of dentate gyrus LTP consolidation is 
mediated by sustained BDNF-TrkB signaling to MNK. Cell Rep. 9, 1430-1445. doi: 10.1016/j.celrep.2014.10.016

Papouin, T., Dunphy, J. M., Tolman, M., Dineley, K. T., and Haydon, P. G. (2017). Septal cholinergic neuromodulation tunes the astrocyte-dependent gating of hippocampal NMDA receptors to wakefulness. Neuron 94, 840-54.e7. doi: 10.1016/j.neuron.2017.04.021

Park, H., Han, K. S., Seo, J., Lee, J., Dravid, S. M., Woo, J., et al. (2015). Channel-mediated astrocytic glutamate modulates hippocampal synaptic plasticity by activating postsynaptic NMDA receptors. Mol. Brain. 8:7. doi: 10.1186/s13041-015-0097-y

Pasti, L., Volterra, A., Pozzan, T., and Carmignoto, G. (1997). Intracellular calcium oscillations in astrocytes: a highly plastic, bidirectional form of communication between neurons and astrocytes in situ. J. Neurosci. 17, 7817-7830. doi: 10.1523/JNEUROSCI.17-20-07817.1997

Patel, T. D., Jackman, A., Rice, F. L., Kucera, J., and Snider, W. D. (2000). Development of sensory neurons in the absence of NGF/TrkA signaling in vivo. Neuron 25, 345-357. doi: 10.1016/S0896-6273(00)80899-5

Patrushev, I., Gavrilov, N., Turlapov, V., and Semyanov, A. (2013). Subcellular location of astrocytic calcium stores favors extrasynaptic neuron-astrocyte communication. Cell Calcium 54, 343-349. doi: 10.1016/j.ceca.2013.08.003

Peavy, R. D., and Conn, P. J. (2010). Phosphorylation of mitogenactivated protein kinase in cultured rat cortical glia by stimulation of metabotropic glutamate receptors. J. Neurochem. 71, 603-612. doi: $10.1046 / j .1471-4159.1998 .71020603 . x$

Penagarikano, O. (2007). The pathophysiology of fragile $\mathrm{x}$ syndrome. Ann. Rev. Genom. Hum. Genet. 8:109. doi: 10.1146/annurev.genom.8.080706.092249

Penn, A. C., Zhang, C. L., Georges, F., Royer, L., Reillat, C. B., Hosy, E., et al. (2017). Hippocampal LTP and contextual learning require surface diffusion of AMPA receptors. Nature 549, 384-388. doi: 10.1038/nature23658

Perea, G., and Araque, A. (2005). Properties of synaptically evoked astrocyte calcium signal reveal synaptic information processing by astrocytes. J. Neurosci. 25, 2192-2203. doi: 10.1523/JNEUROSCI.3965-04.2005

Perea, G., and Araque, A. (2007). Astrocytes potentiate transmitter release at single hippocampal synapses. Science 317, 1083-1086. doi: 10.1126/science. 1144640

Perea, G., Gómez, R., Mederos, S., Covelo, A., Ballesteros, J. J., Schlosser, L., et al. (2016). Activity-dependent switch of GABAergic inhibition into glutamatergic excitation in astrocyte-neuron networks. Elife 5:e20362. doi: $10.7554 /$ eLife. 20362

Perez, E. J., Tapanes, S. A., Loris, Z. B., Balu, D. T., and Liebl, D. J. (2017). Enhanced astrocytic d-serine underlies synaptic damage after traumatic brain injury. $J$. Clin. Investig. 127, 3114-3125. doi: 10.1172/JCI92300

Petralia, R. S., Wang, Y. X., Niedzielski, A. S., and Wenthold, R. J. (1996). The metabotropic glutamate receptors, mGluR2 and mGluR3, show unique postsynaptic, presynaptic and glial localizations. Neuroscience 71, 949-976. doi: 10.1016/0306-4522(95)00533-1

Petravicz, J., Fiacco, T. A., and Mccarthy, K. D. (2008). Loss of IP3 receptordependent $\mathrm{Ca} 2+$ increases in hippocampal astrocytes does not affect baseline CA1 pyramidal neuron synaptic activity. J. Neurosci. 28:4967. doi: 10.1523/JNEUROSCI.5572-07.2008

Pfeiffer, B. E., and Huber, K. M. (2009). The state of synapses in fragile X syndrome. Neurosci. Rev. J. Bring. Neurobiol. Neurol. Psychiatry 15, 549-567. doi: $10.1177 / 1073858409333075$

Pin, J. P., and Duvoisin, R. (1995). The metabotropic glutamate receptors: structure and functions. Neuropharmacology 34, 1-26. doi: 10.1016/0028-3908(94)00129-G

Pinheiro, P. S., Perrais, D., Coussen, F., Barhanin, J., Bettler, B., Mann, J. R., et al. (2007). GluR7 is an essential subunit of presynaptic kainate autoreceptors at hippocampal mossy fiber synapses. Proc. Nat. Acad. Sci. 104, 12181-12186. doi: $10.1073 /$ pnas. 0608891104

Pyka, M., Wetzel, C., Aguado, A., Geissler, M., Hatt, H., and Faissner, A. (2011). Chondroitin sulfate proteoglycans regulate astrocytedependent synaptogenesis and modulate synaptic activity in primary embryonic hippocampal neurons. Eur. J. Neurosci. 33, 2187-2202. doi: 10.1111/j.1460-9568.2011.07690.x

Qiu, Z., Sylwestrak, E. L., Lieberman, D. N., Zhang, Y., Liu, X. Y., and Ghosh, A. (2012). The rett syndrome protein MeCP2 regulates synaptic scaling. J. Neurosci. 32, 989-994. doi: 10.1523/JNEUROSCI.0175-11.2012
Ribeiro, F. M., Pires, R., and Ferguson, S. (2011). Huntington's disease and group I metabotropic glutamate receptors. Mol. Neurobiol. 43, 1-11. doi: 10.1007/s12035-010-8153-1

Risher, W. C., Patel, S., Kim, I. H., Uezu, A., Bhagat, S., Wilton, D. K., et al. (2014). Astrocytes refine cortical connectivity at dendritic spines. Elife 3:e04047. doi: 10.7554/eLife.04047

Rojas, A., Wetherington, J., Shaw, R., Serrano, G., Swanger, S., and Dingledine, R. (2013). Activation of group I metabotropic glutamate receptors potentiates heteromeric kainate receptors. Mol. Pharmacol. 83, 106-121. doi: $10.1124 / \mathrm{mol} .112 .081802$

Romano, C., Sesma, M. A., Mcdonald, C. T., O'Malley, K., Pol, A., and Olney, J. W. (1995). Distribution of metabotropic glutamate receptor mGluR5 immunoreactivity in rat brain. J. Comp. Neurol. 355, 455-469. doi: $10.1002 /$ cne. 903550310

Rösch, H., Schweigreiter, R., Bonhoeffer, T., Barde, Y. A., and Korte, M. (2005). The neurotrophin receptor p75NTR modulates long-term depression and regulates the expression of AMPA receptor subunits in the hippocampus. Proc. Natl. Acad. Sci. U. S. A. 102, 7362-7367. doi: 10.1073/pnas. 0502460102

Rosenberg, N., Gerber, U., and Ster, J. (2016). Activation of group II metabotropic glutamate receptors promotes LTP induction at schaffer collateral-CA1 pyramidal cell synapses by priming NMDA receptors. J. Neurosci. 36, 11521-11531. doi: 10.1523/JNEUROSCI.1519-16.2016

Saez-Orellana, F., Godoy, P. A., Silva-Grecchi, T., Barra, K. M., and Fuentealba, J. Modulation of the neuronal network activity by P2X receptors and their involvement in neurological disorders. Pharmacol. Res. (2015). 101, 109-115. doi: 10.1016/j.phrs.2015.06.009

Sala, C., and Segal, M. (2014). Dendritic spines: the locus of structural and functional plasticity. Physiol. Rev. 94, 141-188. doi: 10.1152 /physrev.00012.2013

Saugstad, J. A., Kinzie, J. M., Shinohara, M. M., Segerson, T. P., and Westbrook, G. L. (1997). Cloning and expression of rat metabotropic glutamate receptor 8 reveals a distinct pharmacological profile. Mol. Pharmacol. 51:119. doi: $10.1124 / \mathrm{mol} .51 .1 .119$

Schafer, D. P., Lehrman, E. K., Kautzman, A. G., Koyama, R., Mardinly, A. R., Yamasaki, R., et al. (2012). Microglia sculpt postnatal neural circuits in an activity and complement-dependent manner. Neuron 74, 691-705. doi: 10.1016/j.neuron.2012.03.026

Schmitz, D., Mellor, J., and Nicoll, R. A. (2001). Presynaptic kainate receptor mediation of frequency facilitation at hippocampal mossy fiber synapses. Science 291, 1972-1976. doi: 10.1126/science. 1057105

Schools, G. P., and Kimelberg, H. K. (2015). mGluR3 and mGluR5 are the predominant metabotropic glutamate receptor mRNAs expressed in hippocampal astrocytes acutely isolated from young rats. J. Neurosci. Res. 58, 533-543. doi: 10.1002/(SICI) 1097-4547(19991115)58:4<533::AID-JNR6>3.0.CO;2-G

Schwarz, Y., Oleinikov, K., Schindeldecker, B., Wyatt, A., Weissgerber, P., Flockerzi, V., et al. (2019). TRPC channels regulate Ca2+-signaling and short-term plasticity of fast glutamatergic synapses. PLoS Biol. 17:e3000445. doi: 10.1371/journal.pbio.3000445

Scimemi, A. (2014). Structure, function, and plasticity of GABA transporters. Front. Cell. Neurosci. 8:161. doi: 10.3389/fncel.2014.00161

Servitja, J. M., Masgrau, R., Sarri, E., and Picatoste, F. (2001). Group I metabotropic glutamate receptors mediate phospholipase D stimulation in rat cultured astrocytes. J. Neurochem. 72, 1441-1447. doi: 10.1046/j.1471-4159.1999.721441.x

Shigemoto, R., Abe, T., Nomura, S., Nakanishi, S., and Hirano, T. (1994). Antibodies inactivating mGluR1 metabotropic glutamate receptor block long-term depression in cultured Purkinje cells. Neuron 12, 1245-1255. doi: 10.1016/0896-6273(94)90441-3

Shigemoto, R., Nomura, S., Ohishi, H., Sugihara, H., Nakanishi, S., and Mizuno, N. (1993). Immunohistochemical localization of a metabotropic glutamate receptor, mGluR5, in the rat brain. Neurosci. Lett. 163, 53-57. doi: 10.1016/0304-3940(93)90227-C

Singh, S. K., Stogsdill, J. A., Pulimood, N. S., Dingsdale, H., Kim, Y. H., Pilaz, L. J., et al. (2016). Astrocytes assemble thalamocortical synapses by bridging NRX1alpha and NL1 via hevin. Cell 164, 183-196. doi: 10.1016/j.cell.2015.11.034 
Skowrońska, K., Obara-Michlewska, M., Zielińska, M., and Albrecht, J. (2019). NMDA receptors in astrocytes: in search for roles in neurotransmission and astrocytic homeostasis. Int. J. Mol. Sci. 20:309. doi: 10.3390/ijms20020309

Sladeczek, F., Pin, J. P., Récasens, M., Bockaert, J., Weiss, S., Sladeczek, F., et al. (1985). Glutamate stimulates inositol phosphate formation in striatal neurons. Nature 317, 717-719. doi: 10.1038/317717a0

Snyder, E. M., Philpot, B. D., Huber, K. M., Xin, D., and Bear, M. F. (2001). Internalization of ionotropic glutamate receptors in response to mGluR activation. Nat. Neurosci. 4, 1079-1085. doi: 10.1038/nn746

Sonntag, M., Blosa, M., Schmidt, S., Reimann, K., Blum, K., Eckrich, T., et al. (2018). Synaptic coupling of inner ear sensory cells is controlled by brevicanbased extracellular matrix baskets resembling perineuronal nets. BMC Biol. 16:99. doi: 10.1186/s12915-018-0566-8

Sorg, B. A., Berretta, S., Blacktop, J. M., Fawcett, J. W., Kitagawa, H., Kwok, J. C., et al. (2016). Casting a wide net: role of perineuronal nets in neural plasticity. J. Neurosci. 36, 11459-11468. doi: 10.1523/JNEUROSCI.2351-16.2016

Srinivasan, R., Huang, B. S., Venugopal, S., Johnston, A. D., Chai, H., Zeng, $\mathrm{H}$., et al. (2015). Ca2+ signaling in astrocytes from Ip3r2 -/- mice in brain slices and during startle responses in vivo. Nat. Neurosci. 18, 708-717. doi: $10.1038 / \mathrm{nn} .4001$

Steinhäuser, C., and Gallo, V. (1996). News on glutamate receptors in glial cells. Trends Neurosci. 19, 339-345. doi: 10.1016/0166-2236(96)10043-6

Straub, C., Hunt, D. L., Yamasaki, M., Kim, K. S., Watanabe, M., Castillo, P. E., et al. (2011). Distinct functions of kainate receptors in the brain are determined by the auxiliary subunit Neto1. Nat. Neurosci. 14, 866-873. doi: 10.1038/nn.2837

Sugiyama, H., Ito, I., and Hirono, C. (1987). A new type of glutamate receptor linked to inositol phospholipid metabolism. Nature 325, 531-533. doi: 10.1038/325531a0

Suttkus, A., Morawski, M., and Arendt, T. (2016). Protective properties of neural extracellular matrix. Mol. Neurobiol. 53, 73-82, doi: 10.1007/s12035-014-8990-4

Suvrathan, A., Hoeffer, C. A., Wong, H., Klann, E., and Chattarii, S. (2010). Characterization and reversal of synaptic defects in the amygdala in a mouse model of fragile X syndrome. Proc. Nat. Acad. Sci. 107, 11591-11596. doi: $10.1073 /$ pnas. 1002262107

Sylwestrak, E. L., and Ghosh, A. (2012). Elfn1 regulates target-specific release probability at CA1-interneuron synapses. Science 338, 536-540. doi: $10.1126 /$ science. 1222482

Tanabe, Y., Nomura, A., Masu, M., Shigemoto, R., and Nakanishi, S. (1993). Signal transduction, pharmacological properties, and expression patterns of two rat metabotropic glutamate receptors, mGluR3 and mGluR4. J. Neurosci. 13, 1372-1378. doi: 10.1523/JNEUROSCI.13-04-01372.1993

Tao, W., Ma, C., Bemben, M. A., Li, K. H., Burlingame, A. L., Zhang, M., et al. (2019). Mechanisms underlying the synaptic trafficking of the glutamate delta receptor GluD1. Mol. Psychiatry. 24, 1451-1460. doi: 10.1038/s41380-019-0378-4

Tao-Cheng, J. H., Crocker, V. T., Winters, C. A., Azzam, R., Chludzinski, J., and Reese, T. S. (2011). Trafficking of AMPA receptors at plasma membranes of hippocampal neurons. J. Neurosci. 31:4834. doi: 10.1523/JNEUROSCI.4745-10.2011

Tejada-Simon, M. V. (2015). Modulation of actin dynamics by Racl to target cognitive function. J. Neurochem. 133, 767-779. doi: 10.1111/jnc. 13100

Testa, C., Standaert, D., Young, A., and Penney, J. (1994). Metabotropic glutamate receptor mRNA expression in the basal ganglia of the rat. J. Neurosci. 14:3005. doi: 10.1523/JNEUROSCI.14-05-03005.1994

Vardjan, N., Verkhratsky, A., and Zorec, R. (2017). Astrocytic pathological calcium homeostasis and impaired vesicle trafficking in neurodegeneration. Int. J. Mol. Sci. 18:358. doi: 10.3390/ijms18020358

Vargas, D. L., Nascimbene, C., Krishnan, C., Zimmerman, A. W., and Pardo, C. A. (2010). Neuroglial activation and neuroinflammation in the brain of patients with autism. Dkgest World Latest Med Inform. 57, 67-81. doi: 10.1002/ana.20315

Vesikansa, A., Sallert, M., Taira, T., and Lauri, S. E. (2007). Activation of kainate receptors controls the number of functional glutamatergic synapses in the area CA1 of rat hippocampus. J. Physiol. 583, 145-157. doi: $10.1113 /$ jphysiol.2007.133975
Vignes, M., Clarke, V., Parry, M. J., Bleakman, D., and Collingridge, G. L. (1998). The GluR5 subtype of kainate receptor regulates excitatory synaptic transmission in areas $\mathrm{CA} 1$ and $\mathrm{CA} 3$ of the rat hippocampus. Neuropharmacology 37:1269. doi: 10.1016/S0028-3908(98)00148-8

Wang, S. J., and Gean, P. W. (1999). Long-term depression of excitatory synaptic transmission in the rat amygdala. J Neurosci. 19, 10656-10663. doi: 10.1523/JNEUROSCI.19-24-10656.1999

Wisniewski, K. E., Segan, S. M., Miezejeski, C. M., Sersen, E. A., and Rudelli, R. D. (2010). The $\operatorname{Fra}(\mathrm{X})$ syndrome: neurological, electrophysiological, and neuropathological abnormalities. Am. J. Med. Genet. Part A 38, 476-480. doi: 10.1002/ajmg.1320380267

Wolosker, H., and Radzishevsky, I. (2013). The serine shuttle between glia and neurons: implications for neurotransmission and neurodegeneration. Biochem. Soc. Trans. 41, 1546-1550. doi: 10.1042/BST20130220

Woo, D. H., Han, K. S., Shim, J. W., Yoon, B. E., Kim, E., Bae, J. Y., et al. (2012). TREK-1 and Best1 channels mediate fast and slow glutamate release in astrocytes upon GPCR activation. Cell 151, 25-40. doi: 10.1016/j.cell.2012.09.005

$\mathrm{Xu}, \mathrm{N}$. J., and Henkemeyer, M. (2012). Ephrin reverse signaling in axon guidance and synaptogenesis. Semin. Cell Dev. Biol. 23, 58-64. doi: $10.1016 /$ j.semcdb.2011.10.024

Yamashita, T., Tucker, K. L., and Barde, Y. A. (1999). Neurotrophin binding to the p75 receptor modulates Rho activity and axonal outgrowth. Neuron 24, 585-593. doi: 10.1016/S0896-6273(00)81114-9

Yang, J., Yang, H., Liu, Y., Li, X., Qin, L., Lou, H., et al. (2016). Astrocytes contribute to synapse elimination via type 2 inositol 1,4,5-trisphosphate receptor-dependent release of ATP. Elife 5:e15043. doi: 10.7554/eLife.15043

Yeiser, C. E. (2004). Neurotrophin signaling through the p75 receptor is deficient in traf6-/- mice. J. Neurosci. 24:10521. doi: 10.1523/JNEUROSCI.1390-04.2004

Yong, A. J. H., Tan, H. L., Zhu, Q., Bygrave, A. M., Johnson, R. C., and Huganir, R. L. (2020). Tyrosine phosphorylation of the AMPA receptor subunit GluA2 gates homeostatic synaptic plasticity. Proc. Natl. Acad. Sci. U. S. A. 117, 4948-4958. doi: 10.1073/pnas.1918436117

Yuji, Y., Tetsuya, K., Shunji, Y., Hiroyuki, F., Tatsuya, M., Miyuki, M., et al. (2008). GABAA receptor-mediated acceleration of aging-associated memory decline in APP/PS1 mice and its pharmacological treatment by picrotoxin. PLoS ONE 3:e3029. doi: 10.1371/journal.pone.0003029

Zeidán-Chuliá, F., Rybarczyk-Filho, J. L., Salmina, A. B., de Oliveira, B. H., Noda, M., and Moreira, J. C. (2013). Exploring the multifactorial nature of autism through computational systems biology: calcium and the Rho GTPase RAC1 under the spotlight. Neuromol. Med. 15, 364-383. doi: 10.1007/s12017-013-8224-3

Zhang, L., and Alger, B. E. (2010). Enhanced endocannabinoid signaling elevates neuronal excitability in fragile X syndrome. J. Neurosci. 30:5724. doi: 10.1523/JNEUROSCI.0795-10.2010

Zhuang, Z., Huang, J., Cepero, M. L., and Liebl, D. J. (2011). Eph signaling regulates gliotransmitter release. Commun. Integr. Biol. 4, 223-226. doi: $10.4161 /$ cib.4.2.14507

Conflict of Interest: The authors declare that the research was conducted in the absence of any commercial or financial relationships that could be construed as a potential conflict of interest.

Publisher's Note: All claims expressed in this article are solely those of the authors and do not necessarily represent those of their affiliated organizations, or those of the publisher, the editors and the reviewers. Any product that may be evaluated in this article, or claim that may be made by its manufacturer, is not guaranteed or endorsed by the publisher.

Copyright (ङ 2021 Liu, Ying, Wang, Zheng, Zhao, Yoon, Yu, Yang, Fang and Hua. This is an open-access article distributed under the terms of the Creative Commons Attribution License (CC BY). The use, distribution or reproduction in other forums is permitted, provided the original author (s) and the copyright owner(s) are credited and that the original publication in this journal is cited, in accordance with accepted academic practice. No use, distribution or reproduction is permitted which does not comply with these terms. 